\title{
Léonard de Vinci et le tracé des formes elliptiques
}

\section{Leonardo da Vinci and the drawing of elliptical shapes}

\author{
Jean-Pierre Crettez ${ }^{1}$ \\ ${ }^{1}$ Chercheur émérite à Telecom Paris
}

RÉSUMÉ. Léonard de Vinci nous a laissé, dans les folios du Codex Atlanticus, trois dessins proposant des méthodes de construction des formes elliptiques. L'analyse de ces dessins montre que ces méthodes sont géométriquement correctes et qu'elles sont devenues de plus en plus efficaces.

ABSTRACT. Leonardo da Vinci left us, in the folios of the Atlanticus Codex, three drawings proposing methods of construction of elliptical shapes. Analysis of these drawings shows that these methods are geometrically correct and that they have become increasingly effective.

MOTS-CLÉS. compas à coniques, construction interne, forme elliptique, excentricité, forme première, géométrie interne, maillage carré, maillage harmonique.

KEYWORDS. conical compass, internal construction, elliptical shape, eccentricity, first shape, internal geometry, square mesh, harmonic mesh.

\section{Introduction}

Pour Léonard de Vinci, les phénomènes naturels sont régis par des lois d'essence mathématique. Poussé par sa curiosité scientifique, il a probablement observé que l'ombre d'une pièce d'un florin éclairée par le soleil prenait la forme d'une ellipse, et que lorsqu'il tournait la pièce, la forme elliptique de l'ombre devenait plus étroite. Bien plus, s'il inclinait la surface sur laquelle l'ombre se déposait, la forme elliptique de l'ombre s'agrandissait avec l'inclinaison. La première de ces deux observations représente la projection géométrique sur un plan horizontal d'un cercle situé dans un plan incliné, et l'autre celle d'un cercle plat sur une surface oblique. Ces observations d'un phénomène naturel ${ }^{1}$ correspondent bien à son approche mathématique de la physique. "Où l'on ne peut appliquer aucune des sciences mathématiques, ni aucune de celles qui sont fondées sur les sciences mathématiques, il n'est point de certitude ${ }^{2} . "$

Dans cette approche mathématique, Léonard est persuadé que les formes qui nous entourent sont le résultat géométrique de forces créées par la nature. Le peintre, amené à recréer ces formes, doit comprendre leur formation. "Comprendre le monde, mais aussi le représenter, c'est dès lors comprendre et représenter son rythme et les lois qui l'organisent". "... celui qui apprend la peinture doit posséder des connaissances mathématiques. Cette façon de penser qui est très éloignée de la nôtre, mais qui était fondamentale au temps de la Renaissance $e^{4}$."

Pour représenter ces formes, Léonard a préféré les formes elliptiques aux formes circulaires. En effet le cercle présente une courbure unique, tandis que les arcs d'ellipse possédent une courbure qui varie d'un point à un autre, pouvant mieux s'adapter géométriquement aux contours (ou aux portions de contour) des formes naturelles. Néanmoins, même si le nombre d'ellipses est infini, et si chacune offre une excentricité différente, Léonard ne s'est intéressé qu'à un petit nombre d'entre elles, comme nous verrons plus loin.

\footnotetext{
${ }^{1}$ Ce phénomène appelé l’ombre au flambeau de la géométrie descriptive.

${ }^{2}$ Léonard de Vinci, Manuscrit G, $96 \mathrm{v}$.

${ }^{3}$ D. Arasse : [1], p. 110

${ }^{4}$ K. Clarck[2], p.160 
Nous avons remarqué son approche, lors de l'analyse géométrique interne ${ }^{5}$ de 5 portraits féminins peints par Léonard, mais aussi de celle du Salvator Mundi'. Cette analyse a montré que certaines parties du contour de la tête ou du visage sont assimilables à des arcs d'ellipse.

Récemment, l'étude d'un simple dessin ${ }^{7}$ de Léonard, a démontré que ces arcs d'ellipse appartiennent à une forme globale que nous avons appelée : forme première.

La forme première peut être considérée comme la représentation géométrique de l'esquisse du contour de la tête. Léonard assimile cette courbe fermée à une ellipse. Elle suggère au spectateur l'aspect global de la tête. Son centre fixe sa position, le grand axe indique l'inclinaison (la tête est droite ou penchée) et l'excentricité indique si la tête est plutôt arrondie ou allongée, ou si elle se présente de profil, de trois quarts ou de face. Les dimensions du petit et du grand axes donnent la taille du portrait. C'est précisément à partir de cette forme première que l'artiste peut, grâce à la géométrie interne, établir la formation du profil ou du portrait.

Ainsi, pour effectuer un portrait, Léonard commençait par tracer l'ellipse constituant la forme première. Comment Léonard de Vinci traçait-il les formes elliptiques ? Le tracé des ellipses peut s'effectuer de deux manières : soit en déterminant la position exacte de certains points de la courbe, avant de les relier à main levée ; soit par un tracé continu à l'aide d'un dispositif particulier. Léonard nous a transmis dans le Codex Atlanticus trois dessins explicitant la construction géométrique des ellipses par points : le folio 318 (figure 1), le folio 602, et le folio 1032, mais aussi un dessin concernant le tracé continu, le folio 1093 expliquant le fonctionnement d'un compas parabolique.

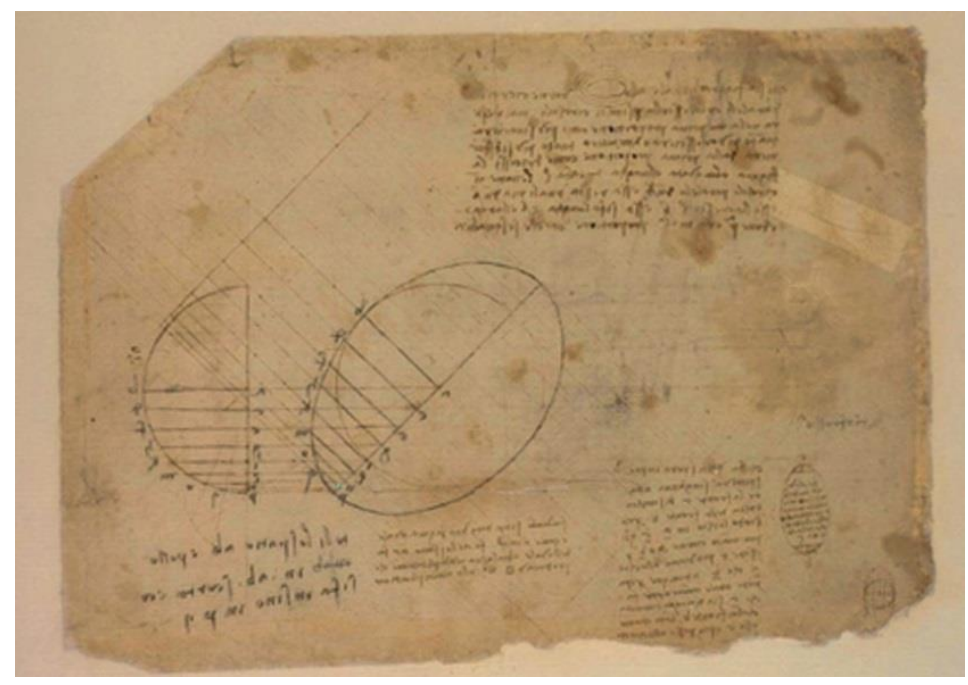

Figure 1. Folio 318 du Codex Atlanticus

\section{L’ellipse : définitions, propriétés et méthodes de construction}

Avant d'analyser ces dessins de Léonard, il convient de revenir sur les définitions des ellipses, leurs constructions et certaines de leurs propriétés. Les ellipses font partie de la famille des coniques dont les premières études furent initiées par Ménechme ${ }^{8}$. Elles furent complétées et approfondies par Apollonius de Perge ${ }^{9}$ dans son traité sur les coniques. Il existe plusieurs définitions de l'ellipse.

\footnotetext{
${ }^{5}$ Crettez J-P.,[3], §. 8.2.3

${ }^{6}$ Crettez J-P.,[4]

${ }^{7}$ Crettez J-P.,[5]

${ }^{8}$ Ménechme (-375 -325) disciple de Platon.

${ }^{9}$ Apollonius de Perge (-262 -190) 


\subsection{Première définition de l'ellipse : Df1}

L'ellipse est le lieu des points du plan dont les distances à un point fixe $\boldsymbol{F}$ (le foyer) et à une droite (D) (la directrice) sont dans un rapport constant, appelé l'excentricité : $\mathbf{M F} / \mathbf{M K}=\mathbf{\varepsilon}$. L'excentricité $\boldsymbol{\varepsilon}$, comprise entre 0 et 1 , est caractéristique de la forme de l'ellipse (figure 2).

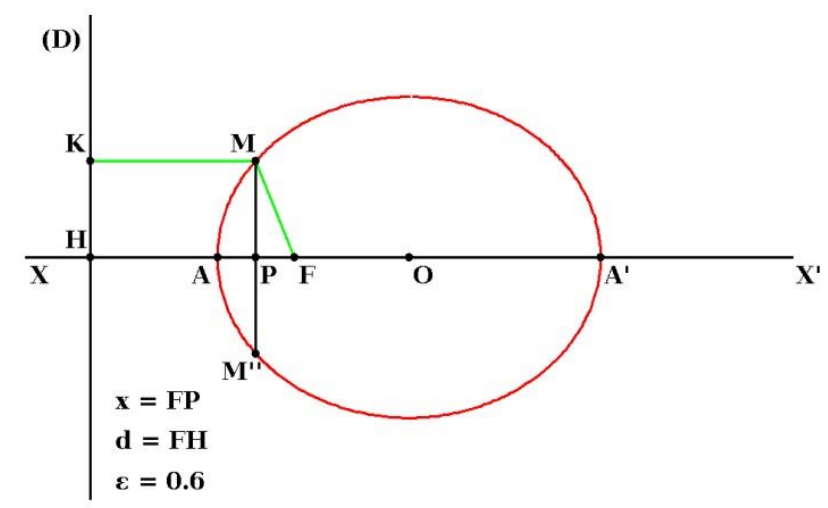

Figure 2. Lieu des points dont le rapport des distances à un point fixe et à une droite fixe est constant

Soient $\mathbf{F}$ le foyer, (D) la directrice et $\boldsymbol{\varepsilon}$ l'excentricité, $\mathbf{H}$ le pied de la perpendiculaire menée du foyer $\mathbf{F}$ à la droite (D), et soit $\mathbf{d}$ est la distance de $\mathbf{F}$ à $\mathbf{H}$. Ces deux points sont situés sur l'axe focal XX'. M se projette sur cet axe au point $\mathbf{P}$. Cet axe est un axe de symétrie, car la définition sousentend l'existence d'un point M' symétrique de $\mathbf{M}$, se projetant lui aussi au point P. Si F est l'origine des abscisses dirigées positivement de $\mathbf{F}$ vers $\mathbf{H}$, l'abscisse de $\mathbf{M}$ est $\mathbf{x}=\overline{\mathbf{F P}}$.

L'ordonnée $\mathbf{y}$ de $\mathbf{M}$ est telle que $: \mathbf{y}^{2}=\overline{\mathbf{M P}}^{2}=\overline{\mathbf{M F}}^{2}-\mathbf{x}^{2}=\boldsymbol{\varepsilon}^{2}(\mathbf{d}-\mathbf{x})^{2}-\mathbf{x}^{2}=(\boldsymbol{\varepsilon}(\mathbf{d}-\mathbf{x})-\mathbf{x}) \cdot(\boldsymbol{\varepsilon}(\mathbf{d}-\mathbf{x})+\mathbf{x})$. Son carré s'annule pour 2 valeurs de $\mathbf{x}: \mathbf{x}_{\mathbf{A}}=\mathbf{d} \boldsymbol{\varepsilon} /(\boldsymbol{\varepsilon}+\mathbf{1})$ et $\mathbf{x}_{\mathbf{A}},=\mathbf{d} \boldsymbol{\varepsilon} /(\boldsymbol{\varepsilon}-\mathbf{1})$. Ces valeurs correspondent aux abscisses des points $\mathbf{A}$ et $\mathbf{A}^{\prime}$, sommets de l'ellipse.

Le segment $\overline{\mathbf{A}^{\prime} \mathbf{A}}$ a pour longueur $\mathbf{x}_{\mathbf{A}^{-}}-\mathbf{x}_{\mathbf{A}},=\mathbf{2 d} \boldsymbol{\varepsilon} /\left(\mathbf{1}-\boldsymbol{\varepsilon}^{\mathbf{2}}\right)$, par suite le demi-grand axe a de l'ellipse a pour valeur $\mathbf{a}=\mathbf{\varepsilon d} /\left(\mathbf{1}-\boldsymbol{\varepsilon}^{\mathbf{2}}\right)$. Son centre $\mathbf{O}$ est tel que $\overline{\mathbf{F O}}=(\overline{\mathbf{F A}}+\overline{\mathbf{F A}}) / \mathbf{2}=-\mathbf{d} \boldsymbol{\varepsilon}^{\mathbf{2}} /\left(\mathbf{1}-\boldsymbol{\varepsilon}^{\mathbf{2}}\right)$. La distance focale $\mathbf{f}$ de l'ellipse a pour valeur $\mathbf{f}=\mathbf{d} \boldsymbol{\varepsilon}^{2} /\left(\mathbf{1}-\boldsymbol{\varepsilon}^{\mathbf{2}}\right)$. Le carré de l'ordonnée $\mathbf{y}$ de $\mathbf{M}$ peut alors s'écrire :

$$
\mathbf{y}^{2}=\overline{\mathbf{M P}}^{2}=-\left(1-\varepsilon^{2}\right)\left(x_{\mathrm{A}}-\mathbf{x}\right)\left(\mathbf{x}_{\mathrm{A}},-\mathrm{x}\right)=-\left(1-\varepsilon^{2}\right) \overline{\mathbf{P A}^{\prime}} \cdot \overline{\mathbf{P A}^{\prime}}
$$

\subsection{Deuxième définition de l'ellipse : Df2}

L'ellipse est le lieu des points dont la somme des distances à deux points fixes (les foyers) est constante.

Soit $\mathbf{P}^{\prime}$ le symétrique de $\mathbf{P}$ par rapport au centre $\mathbf{O}$ (figure 3 ). Nous avons $\overline{\mathbf{P}^{\prime} \mathbf{A}^{\prime}}=-\overline{\mathbf{P A}}$ et $\overline{\mathbf{P}^{\prime} \mathbf{A}}=-$ $\overline{\mathbf{P A}^{\prime}}$. Par suite $: \overline{\mathbf{P}^{\prime} \mathbf{A}} \cdot \overline{\mathbf{P}^{\prime} \mathbf{A}^{\prime}}=\overline{\mathbf{P A}} \cdot \overline{\mathbf{P A}}$. Il existe ainsi un point $\mathbf{M}^{\prime}$ qui se projette en $\mathbf{P}^{\prime}$ tel que $: \overline{\mathbf{M}^{\prime} \mathbf{P}^{\prime 2}}=-$ $\left(1-\varepsilon^{2}\right) \overline{\mathbf{P}^{\prime} \mathbf{A}} \cdot \overline{\mathbf{P}^{\prime} \mathbf{A}^{\prime}}=-\left(1-\varepsilon^{2}\right) \overline{\mathbf{P A}} \cdot \overline{\mathbf{P A}^{\prime}}=\overline{\mathbf{M P}^{2}}$

Le point M' d'abscisse P', a même ordonnée que le point $\mathbf{M}$. Il est symétrique de $\mathbf{M}$ par rapport à un axe YY' passant par $\mathbf{O}$ et orthogonal à l'axe $\mathbf{X} \mathbf{X}^{\prime}$ '. La courbe est donc symétrique par rapport à cet axe. Cette symétrie entraîne l'existence d'un foyer $\mathbf{F}$ ' et d'une directrice (D'), symétriques de $\mathbf{F}$ et (D). 


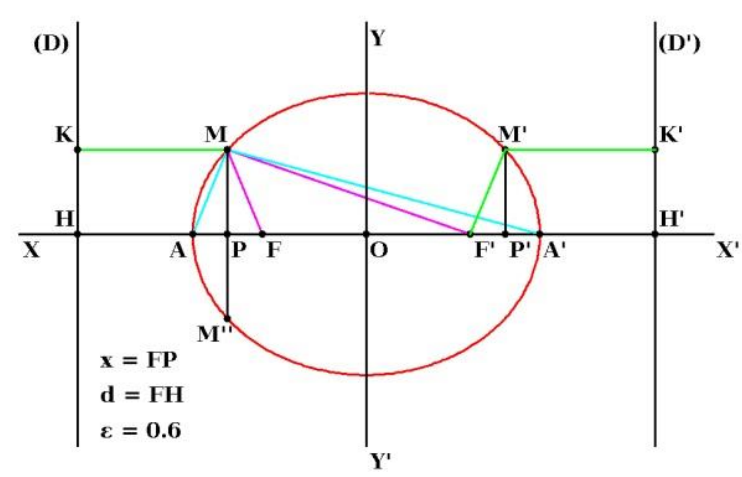

Figure 3. Lieu des points dont la somme des distances à deux points fixes est constante

Soient $\mathbf{K}$ et $\mathbf{K}^{\prime}$ ' les projections de $\mathbf{M}$ et $\mathbf{M}$ ' sur les directrices (D) et (D'). Nous avons $\mathbf{M F}=\mathbf{\varepsilon M K}$ et $\mathbf{M}^{\prime} \mathbf{F}^{\prime}=\mathbf{\varepsilon} \mathbf{M}^{\prime} \mathbf{K}^{\prime}$. Mais quand $\mathbf{M}^{\prime}$ vient en $\mathbf{M}, \mathbf{M F}{ }^{\prime}=\mathbf{\varepsilon M K}$ '. La somme des distances $\mathbf{M F}+\mathbf{M F}$ '= $\boldsymbol{\varepsilon}\left(\mathbf{M K}+\mathbf{M K} \mathbf{K}^{\prime}\right)=\boldsymbol{\varepsilon} \mathbf{K} \mathbf{K}^{\prime}$ est constante. Elle est égale au grand axe de l'ellipse : $\mathbf{A} \mathbf{A}^{\prime}=2 \mathbf{a}$. D'où la deuxième définition de l'ellipse.

L'ellipse peut être simplement construite par la méthode du jardinier, au moyen d'un fil de longueur $2 \mathbf{a}$, tendu par un crayon et dont les extrémités sont fixées aux points $\mathbf{F}$ et $\mathbf{F}$ '.

\subsection{Troisième définition de l'ellipse : Df3}

L'ellipse peut être considérée comme la projection orthogonale sur un plan horizontal, d'un cercle situé dans un plan incliné.

Soit $(\mathbf{C})$ le cercle de centre $\mathbf{O}$ et de diamètre $\mathbf{A A}$ ' (figure 4a). La droite $\mathbf{P M}$ coupe ce cercle en un point $\mathbf{N}$, au-dessus de $\mathbf{M}$, et pour les ordonnées négatives elle coupe l'ellipse en $\mathbf{M}$ ' et le cercle en $\mathbf{N}^{\prime}$. À cause des propriétés du cercle, le carré de l'ordonnée de $\mathbf{N}$ et le carré de l'ordonnée de $\mathbf{N}^{\prime}$ ont pour valeur $\overline{\mathbf{N P}}^{2}={\overline{\mathbf{N}^{\prime}}}^{2}=-\overline{\mathbf{P A}} \cdot \overline{\mathbf{P A}}$.

Le rapport entre les carrés des ordonnées de $\mathbf{M}$ et $\mathbf{N}$ vaut $\overline{\mathbf{M P}}^{2} / \overline{\mathbf{N P}}^{2}=\overline{\mathbf{M}}^{\prime} \mathbf{P}^{2} / \overline{\mathbf{N}} \mathbf{\prime}^{2}=\left(\mathbf{1}-\boldsymbol{\varepsilon}^{2}\right)$. Les ordonnées de $\mathbf{M}$ et de $\mathbf{N}$, et par symétrie celles de $\mathbf{M}^{\prime}$ et $\mathbf{N}^{\prime}$, sont respectivement dans le rapport $\sqrt{\mathbf{1}-\boldsymbol{\varepsilon}^{\mathbf{2}}}$, quelle que soit la position de l'abscisse de $\mathbf{M}$.

En particulier, lorsque le point $\mathbf{P}$ est au point $\mathbf{O}$, le point $\mathbf{M}$ est au maximum : $\mathbf{N P}=\mathbf{a}$, et $\mathbf{M P}=$ b. La valeur de $\mathbf{b}$ est ainsi : $\mathbf{b}=\mathbf{a} \sqrt{\mathbf{1 - \varepsilon ^ { 2 }}}=\mathbf{\varepsilon d} / \sqrt{\mathbf{1}-\boldsymbol{\varepsilon}^{\mathbf{2}}}$.

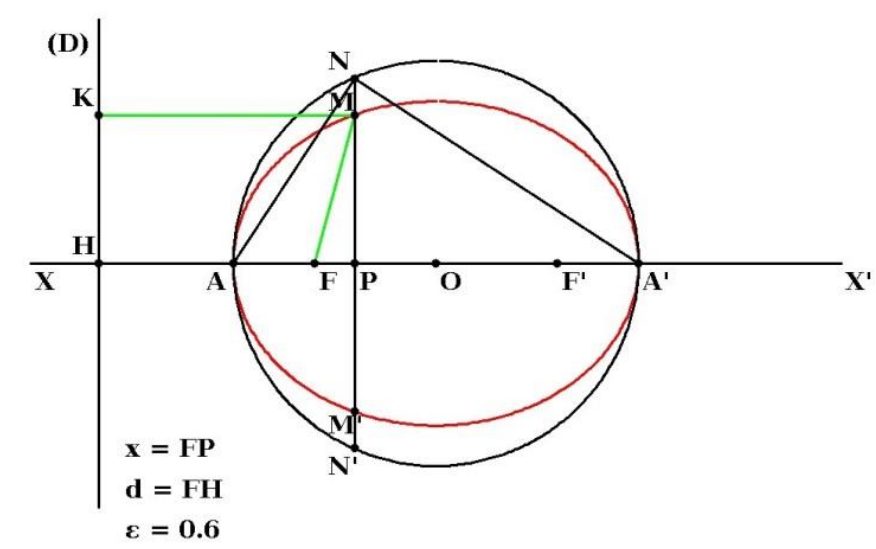

Figure 4a. Le cercle principal de l'ellipse

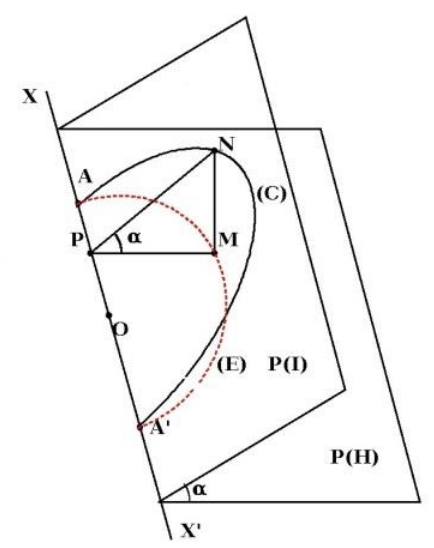

Figure 4b. Projection orthogonale sur un plan horizontal d'un cercle situé dans un plan incliné 
Tout se passe comme si l'ellipse était située dans un plan horizontal $\mathbf{P}(\mathbf{H})$, le cercle dans un plan $\mathbf{P}(\mathbf{I})$ incliné d'un angle $\boldsymbol{\alpha}$ par rapport au plan horizontal, et le coupant suivant l'axe $\mathbf{X X}$ ', et par suite, les points $\mathbf{N}$ du cercle se projetant sur les points $\mathbf{M}$ de l'ellipse (figure $4 b$ ).

$$
\overline{\mathrm{MP}}=\overline{\mathbf{N P}} \sqrt{\mathbf{1 - \varepsilon ^ { 2 }}}=\overline{\mathbf{N P}} \cos (\alpha) \text {, avec } \varepsilon=\sin (\alpha) .
$$

L'excentricité $\boldsymbol{\varepsilon}$ d'une ellipse est simplement égale au sinus de l'angle d'inclinaison $\boldsymbol{\alpha}$ d'un plan sur l'autre.

Plus précisément, si $\left(\mathbf{x}_{\mathbf{c}}, \mathbf{y}_{\mathbf{c}}\right)$ sont les coordonnées de $\mathbf{N}$ dans le plan $\mathbf{P}(\mathbf{I})$ et $\left(\mathbf{x}_{\mathbf{e}}, \mathbf{y}_{\mathbf{e}}\right)$ les coordonnées de $\mathbf{M}$ dans le plan $\mathbf{P}(\mathbf{H}): \mathbf{x}_{\mathbf{e}}=\mathbf{x}_{\mathbf{c}}$ et $\mathbf{y}_{\mathbf{e}}=\mathbf{y}_{\mathbf{c}} \cos (\boldsymbol{\alpha})$. Les abscisses sont identiques, et les ordonnées sont réduites dans le rapport constant $\cos (\boldsymbol{\alpha})$. Par suite, le rapport $\mathbf{r}_{\mathbf{e c}}$ entre la surface de l'ellipse et celle du cercle principal est $\mathbf{r}_{\mathrm{ec}}=\pi \mathbf{a b} / \pi \mathbf{a}^{2}=\mathbf{b} / \mathbf{a}=\cos (\boldsymbol{\alpha})$.

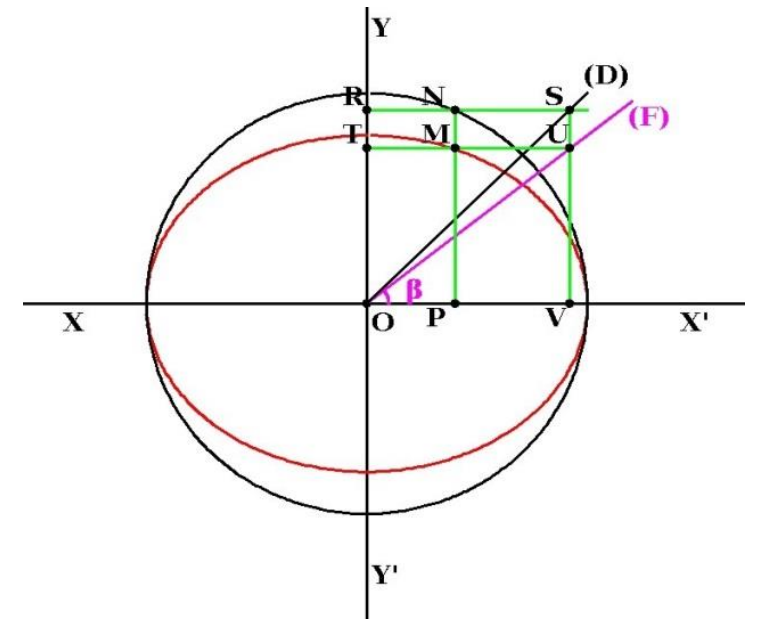

Figure 5a. Construction de l'ellipse par points

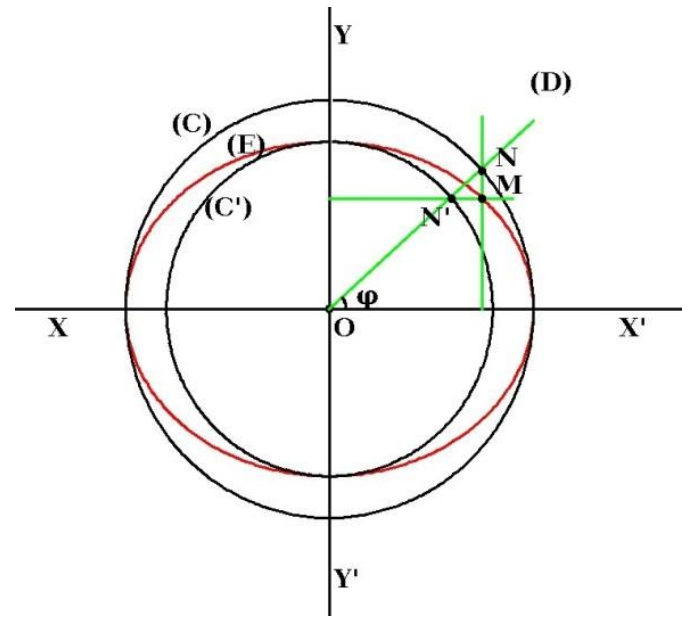

Figure 5b. Construction de l'ellipse à partir des deux cercles principaux

Léonard pouvait construire l'ellipse point par point en associant à chaque point $\mathbf{N}$ du cercle principal un point $\mathbf{M}$ de l'ellipse tel que $\mathbf{y}_{\mathbf{e}}=(\mathbf{b} / \mathbf{a}) \mathbf{y}_{\mathbf{c}}$ : figure 5a. L'ordonnée $\mathbf{y}_{\mathbf{c}}$ de $\mathbf{N}$ est égale à $\mathbf{N P}=$ $\mathbf{S V}$, qui à l'aide de la bissectrice (D) se transforme en une abscisse $\mathbf{x}=\mathbf{S R}=\mathbf{V O}$ pour la fonction (F) telle $\mathbf{y}=\mathbf{b x} / \mathbf{a}$. Cette dernière détermine l'ordonnée $\mathbf{V U}=\mathbf{P M}=\mathbf{y}_{\mathrm{e}}$ du point $\mathbf{M}$.

Une méthode plus élégante consiste à tracer le petit cercle principal $\left(\mathbf{C}^{\prime}\right)$ de rayon $\mathbf{b}$. Pour chaque point $\mathbf{N}$ du cercle $(\mathbf{C})$, le rayon $\mathbf{O N}$ coupe ce cercle en $\mathbf{N}$ ' (figure 5 b) et l'on détermine $\mathbf{M}$ par l'intersection des deux droites perpendiculaires : l'une parallèle à la droite des foyers et passant par $\mathbf{N}$ ', et l'autre parallèle à la directrice et passant par $\mathbf{N}$.

Le point $\mathbf{N}$ a pour coordonnées $\left(\mathbf{x}_{\mathbf{N}}=\operatorname{acos}(\varphi), \mathbf{y}_{\mathbf{N}}=\operatorname{asin}(\varphi)\right)$, le point $\mathbf{N}^{\prime}$ a pour coordonnées $\left(\mathbf{x}_{\mathbf{N}}\right.$, $\left.=\operatorname{bcos}(\varphi), \mathbf{y}_{\mathbf{N}},=\operatorname{bsin}(\varphi)\right)$ et le point $\mathbf{M}$ a pour coordonnées $\left(\mathbf{x}_{\mathrm{M}}=\operatorname{acos}(\varphi), \mathbf{y}_{\mathrm{M}}=\mathbf{b s i n}(\varphi)\right)$.

Le point $\mathbf{M}$ vérifie ainsi la définition algébrique de l'ellipse : $\left(\mathbf{x}_{M} / \mathbf{a}\right)^{2}+\left(\mathbf{y}_{M} / \mathbf{b}\right)^{2}=\mathbf{1}$

\subsection{Quatrième définition de l'ellipse : Df4}

L'ellipse peut être considérée comme la projection orthogonale d'un cercle situé dans un plan horizontal, sur un plan incliné, ou de façon plus imagée, comme l'intersection d'un cylindre par un plan incliné.

Soit un cylindre placé verticalement (figure 6). Sa section droite est un cercle (C) de rayon $\mathbf{r}$, placé dans le plan horizontal $\mathbf{P}(\mathbf{H})$. Le cylindre est coupé par un plan $\mathbf{P}(\mathbf{I})$, incliné d'un angle $\boldsymbol{\alpha}$ par rapport au plan horizontal $\mathbf{P}(\mathbf{H})$. 


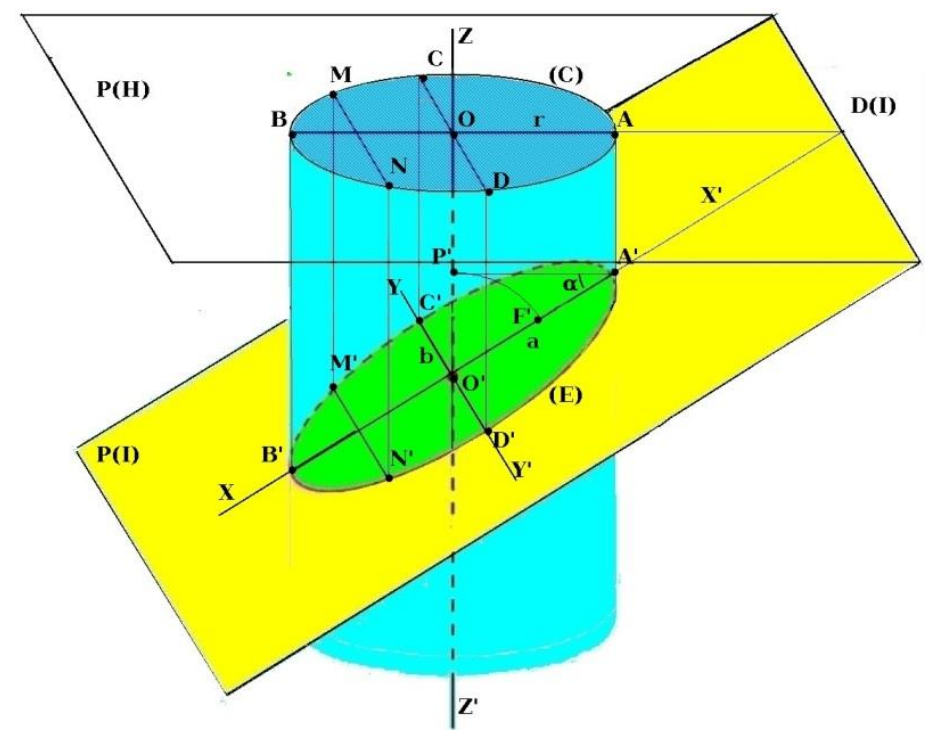

Figure 6. Intersection d'un cylindre par un plan incliné

Toute sécante du cercle $(\mathbf{C})$ parallèle à l'intersection $\mathbf{D}(\mathbf{I})$ des deux plans, détermine un segment de droite qui se projette sur le plan incliné $\mathbf{P}(\mathbf{I})$, suivant un autre segment de droite de même orientation et de même longueur : $\mathbf{M}^{\prime} \mathbf{N}^{\prime}=\mathbf{M N}$. En particulier, le segment de droite $\mathbf{C}^{\prime} \mathbf{D}^{\prime}$, projection du segment de droite CD, passant par le point $\mathbf{O}$ ' de l'axe vertical du cylindre, correspond au petit axe de l'ellipse. Le demi-petit axe b de l'ellipse formé par le segment de droite $\mathbf{O}$ 'C', est donc égal à $\mathbf{r}$.

Toute sécante du cercle $(\mathbf{C})$ perpendiculaire à l'intersection $\mathbf{D}(\mathbf{I})$, détermine un segment de droite qui se projette sur le plan incliné suivant un autre segment de droite de même orientation, mais de plus grande longueur. En particulier, le segment de droite $\mathbf{A}^{\prime} \mathbf{B}^{\prime}$, projection du segment de droite AB, passant par le point $\mathbf{O}^{\prime}$ de l'axe vertical du cylindre, correspond au grand axe de l'ellipse. Le demi-grand axe $\mathbf{a}=\mathbf{O}^{\prime} \mathbf{A}^{\prime}$ est tel que $\mathbf{r} / \mathbf{a}=\cos (\boldsymbol{\alpha})$ soit $\mathbf{a}=\mathbf{b} / \cos (\boldsymbol{\alpha})$.

Plus généralement, si $\left(\mathbf{x}_{\mathbf{c}}, \mathbf{y}_{\mathbf{c}}\right)$ sont les coordonnées de $\mathbf{M}$ d'un point du cercle dans le plan $\mathbf{P}(\mathbf{H})$ et $\left(\mathbf{x}_{\mathbf{e}}, \mathbf{y}_{\mathbf{e}}\right)$ les coordonnées de $\mathbf{M}^{\prime}$ du point correspondant de l'ellipse dans le plan $\mathbf{P}(\mathbf{I}): \mathbf{x}_{\mathbf{e}}=\mathbf{x}_{\mathbf{c}} / \cos (\boldsymbol{\alpha})$ et $\mathbf{y}_{\mathbf{e}}=\mathbf{y}_{\mathbf{c}}$.

Les ordonnées sont identiques, et les abscisses sont augmentées dans le rapport constant $\mathbf{1} / \cos (\boldsymbol{\alpha})$.

Dans le triangle rectangle $\mathbf{O}^{\prime} \mathbf{A}^{\prime} \mathbf{P}^{\prime}$, le segment $\mathbf{O}^{\prime} \mathbf{P}^{\prime}=\sqrt{\mathbf{a}^{2}-\mathbf{b}^{2}}$ est égal à la distance focale $\mathbf{f}$. Par suite l'excentricité $\varepsilon$ de l'ellipse vaut $\varepsilon=\mathbf{f} / \mathbf{a}=\mathbf{O}^{\prime} \mathbf{P}^{\prime} / \mathbf{O}^{\prime} \mathbf{A}^{\prime}=\sin (\boldsymbol{\alpha})$. L'excentricité $\varepsilon$ de l'ellipse est encore liée à l'inclinaison $\boldsymbol{\alpha}$ entre les deux plans. Le cercle inscrit à l'intérieur de l'ellipse est appelé le petit cercle principal. Il a pour rayon $\mathbf{b}=\mathbf{r}$.

Le rapport $\mathbf{r}_{\mathbf{e c}}$ des surfaces de l'ellipse et du cercle est $\mathbf{r}_{\mathbf{e c}}=\pi \mathbf{a b} / \pi \mathbf{b}^{2}=\mathbf{a} / \mathbf{b}=\mathbf{1} / \mathbf{c o s}(\boldsymbol{\alpha})$.

Lorsque $\boldsymbol{\alpha}=0^{\circ}, \boldsymbol{\varepsilon}=0$, les deux plans sont parallèles : $\mathbf{x}_{\mathbf{e}}=\mathbf{x}_{\mathbf{c}}, 1^{\prime}$ 'ellipse est identique au cercle.

Lorsque $\boldsymbol{\alpha}=30^{\circ}, \boldsymbol{\varepsilon}=0.5, \mathbf{x}_{\mathbf{e}}=1,154 \mathbf{x}_{\mathbf{c}}$, la surface est 1,154 fois plus grande

Lorsque $\boldsymbol{\alpha}=36^{\circ} 87, \boldsymbol{\varepsilon}=0.6, \mathbf{x}_{\mathbf{e}}=1.25 \mathbf{x}_{\mathbf{c}}$, la surface est 1,25 fois plus grande

Lorsque $\boldsymbol{\alpha}=45^{\circ}, \boldsymbol{\varepsilon}=0.707, \mathbf{x}_{\mathbf{e}}=1.414 \mathbf{x}_{\mathbf{c}}$, la surface est 1,414 fois plus grande

Lorsque $\boldsymbol{\alpha}=60^{\circ}, \boldsymbol{\varepsilon}=0.866, \mathbf{x}_{\mathbf{e}}=2 \mathbf{x}_{\mathbf{c}}$, la surface est 2 fois plus grande.

Lorsque $\boldsymbol{\alpha} \rightarrow 90^{\circ}, \boldsymbol{\varepsilon} \rightarrow 1$, l'ellipse dégénère en un segment de droite. 


\section{Analyse de trois folios du Codex Atlanticus}

Le Codex Atlanticus est un recueil de dessins et de notes de Léonard de Vinci qui ont été collés et rassemblés après sa mort, en 12 volumes contenant plus de 1119 feuillets (folios), par le sculpteur Pompeo Léoni. Parmi ces feuillets qui couvrent une longue période de sa vie de 1478 à 1518, nous avons remarqué 3 feuillets relatifs à la construction des formes elliptiques par points.

\subsection{Principe de construction de l'ellipse du folio 602}

Le schéma de construction de la forme elliptique représentée sur le folio 602 (1499) du Codex Atlanticus, montre que Léonard a proposé, de façon probablement intuitive, une méthode originale qui s'approche du principe de la projection sur un plan incliné d'un cercle placé dans un plan horizontal : selon la définition Df4. Mais ici, Léonard n'a pas placé le cercle principal et l'ellipse dans l'espace, mais dans un même plan : celui du dessin.

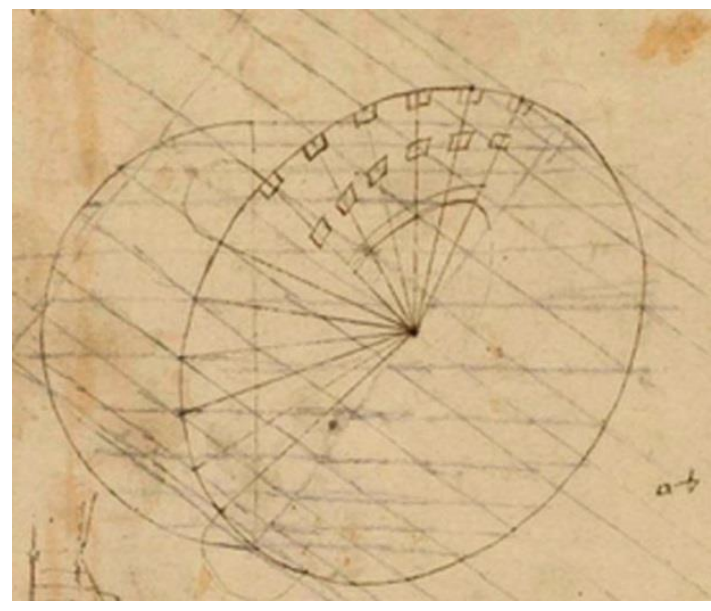

Figure 7. La forme elliptique du folio 602

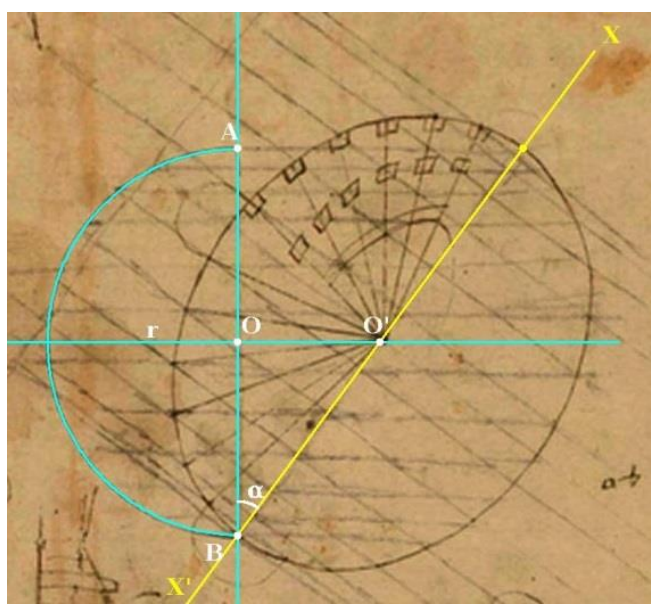

Figure 8. Les bases de sa construction

Le dessin du folio 602 est bien explicite (figure 7). Léonard a tracé (figure 8) un demi-cercle de centre $\mathbf{O}$ et de diamètre $\mathbf{A B}$. Puis il a mené un axe $\mathbf{X} \mathbf{X}^{\prime}$ passant par $\mathbf{B}$, et incliné d'un angle $\boldsymbol{\alpha}$ par rapport à l'axe $\mathbf{A B}$.

(Remarque : curieusement, ni le centre O, ni l'axe OO' ne figurent dans le dessin de Léonard.)

Il a ensuite tracé (figure 9) un faisceau de droites horizontales, différemment espacées, traversant le demi-cercle, en formant des segments de droites qui sont plus rapprochés lorsqu'ils sont près des sommets $\mathbf{A}$ et $\mathbf{B}$. Ce faisceau de droites coupe l'axe (XX') en plusieurs points. Par chacun de ces points, il a fait passer une droite perpendiculaire à cet axe $\left(\mathbf{X X}^{\prime}\right)^{\prime}$, formant ainsi, un autre faisceau de droites parallèles.

Puis, Léonard a reporté à l'aide de l'ouverture d'un compas, et de façon symétrique, chacun des segments de droite définis par le faisceau horizontal, sur la droite correspondante perpendiculaire à l'axe (XX'). Par exemple (figure 9), le segment $\mathbf{M N}$ est reporté selon les segments $\mathbf{M}^{\prime} \mathbf{N}^{\prime}$ et $\mathbf{N}^{\prime} \mathbf{M}^{\prime}$ ' de chaque côté de l'axe (XX').

Il suffit alors de joindre leurs extrémités pour dessiner l'ellipse. Si e est l'espacement entre deux droites horizontales traversant le cercle, et e' l'espacement entre les droites correspondantes de l'ellipse, les deux espacements vérifient la relation : $\mathbf{e}^{\prime}=\mathbf{e} / \mathbf{c o s}(\boldsymbol{\alpha})$. 


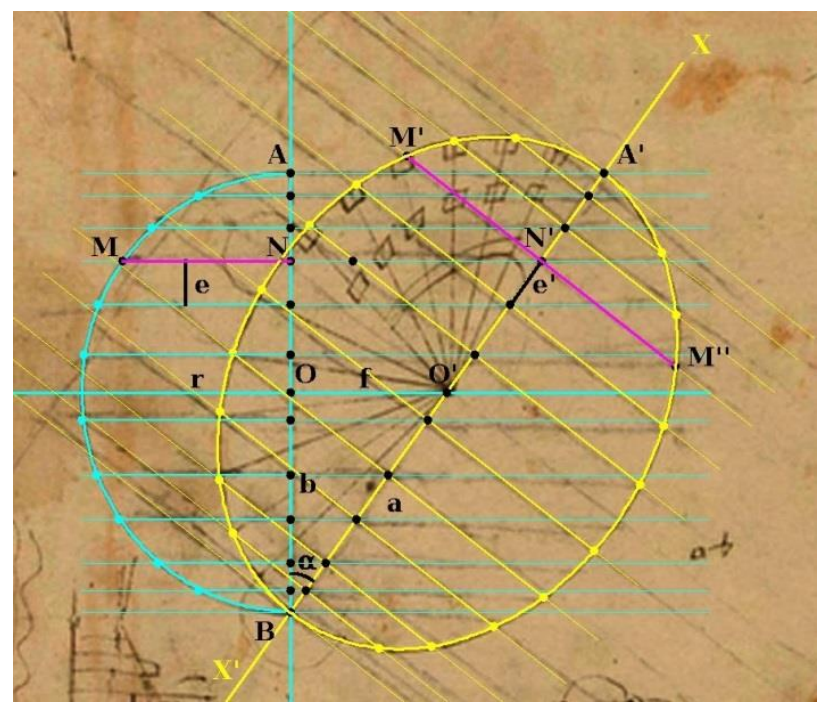

Figure 9. Construction de l'ellipse à partir du demi-cercle principal

Le demi-grand axe $\mathbf{a}=\mathbf{O}^{\prime} \mathbf{A}^{\prime}=\mathbf{O}^{\prime} \mathbf{A}$ est tel que $\mathbf{r} / \mathbf{a}=\cos (\boldsymbol{\alpha})$, soit $\mathbf{a}=\mathbf{r} / \cos (\boldsymbol{\alpha})$.

Plus généralement, soit AB l'axe des abscisses d'un système de coordonnées de centre $\mathbf{O}$, et soit $\mathbf{X X}$ ' l'axe des abscisses d'un autre système de coordonnées de centre $\mathbf{O}$ ', à tout point $\mathbf{M}$ de coordonnées $\left(\mathbf{x}_{\mathbf{c}}, \mathbf{y}_{\mathbf{c}}\right)$ du demi-cercle, la construction fait correspondre un point $\mathbf{M}^{\prime}$ de coordonnées $\left(\mathbf{x}_{\mathbf{e}}, \mathbf{y}_{\mathbf{e}}\right)$ appartenant à l'ellipse, tel que: $\mathbf{y}_{\mathbf{e}}=\mathbf{y}_{\mathbf{c}}$ et $\mathbf{x}_{\mathbf{e}}=\mathbf{x}_{\mathbf{c}} / \cos (\boldsymbol{\alpha})$, et un autre point $\mathbf{M}$ ' de coordonnées

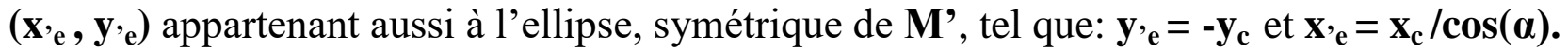

L'ellipse construite par Léonard vérifie bien les propriétés de la projection orthogonale sur un plan incliné, d'un cercle situé dans un plan horizontal, selon la définition Df4.

\subsubsection{Interprétation géométrique de la méthode de Léonard}

Le dessin de Léonard étant tracé dans un plan vertical $\mathbf{P}(\mathbf{V})$, situé dans un espace à trois dimensions (figure 10), nous faisons tourner le cercle principal de $90^{\circ}$ autour de l'axe $\mathbf{A B}$ pour l'insérer dans un plan vertical $\mathbf{P}(\mathbf{C})$ perpendiculaire au plan $\mathbf{P}(\mathbf{V})$, et nous faisons tourner l'ellipse de $90^{\circ}$ autour de l'axe $\mathbf{X X}$ ' pour la mettre dans un plan incliné $\mathbf{P}(\mathbf{E})$ perpendiculaire au plan $\mathbf{P}(\mathbf{V})$. Le cercle correspond ainsi à la section droite d'un cylindre de rayon $\mathbf{r}$ et d'axe horizontal $\mathbf{Z Z}$ '. Le plan $\mathbf{P}(\mathbf{E})$ incliné d'un angle $\boldsymbol{\alpha}$, coupe ce cylindre pour former l'ellipse selon la définition Df4.

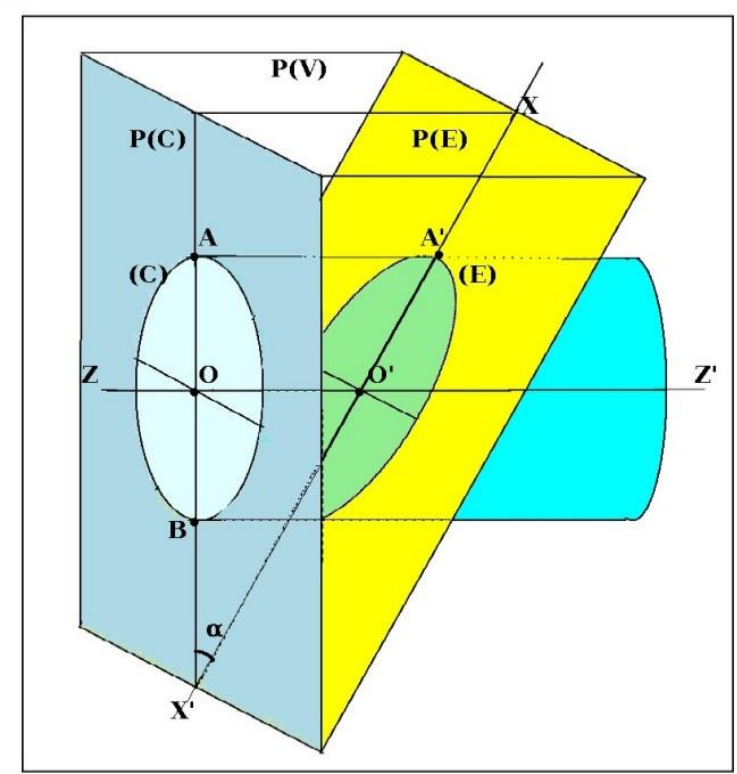

Figure 10. Interprétation géométrique de la méthode décrite par Léonard. 
La démarche de Léonard pour construire cette ellipse correspond bien à la quatrième définition de l'ellipse : Df4, à savoir l'intersection d'un cylindre par un plan incliné d'un angle $\boldsymbol{\alpha}$ par rapport à la section droite. La forme de l'ellipse est indépendante de l'emplacement où a lieu l'intersection. Même si le report des segments de droite est fastidieux, la méthode de Léonard est correcte : elle est d'autant plus précise que le nombre de droites traversant le demi-cercle est élevé.

\subsubsection{Choix de l'ellipse du folio 602 et sa construction dans un maillage carré}

Pour dessiner l'ellipse du folio 602, Léonard a choisi une ellipse particulière. Il l'a construite à partir de deux éléments : le diamètre du demi-cercle et l'angle d'inclinaison $\boldsymbol{\alpha}$. Ces deux éléments sont contenus dans le triangle rectangle $\mathbf{A A}$ 'B (figure 11). Ce triangle choisi par Léonard est un triangle de Pythagore : ses côtés sont proportionnels à 3, 4, 5 .

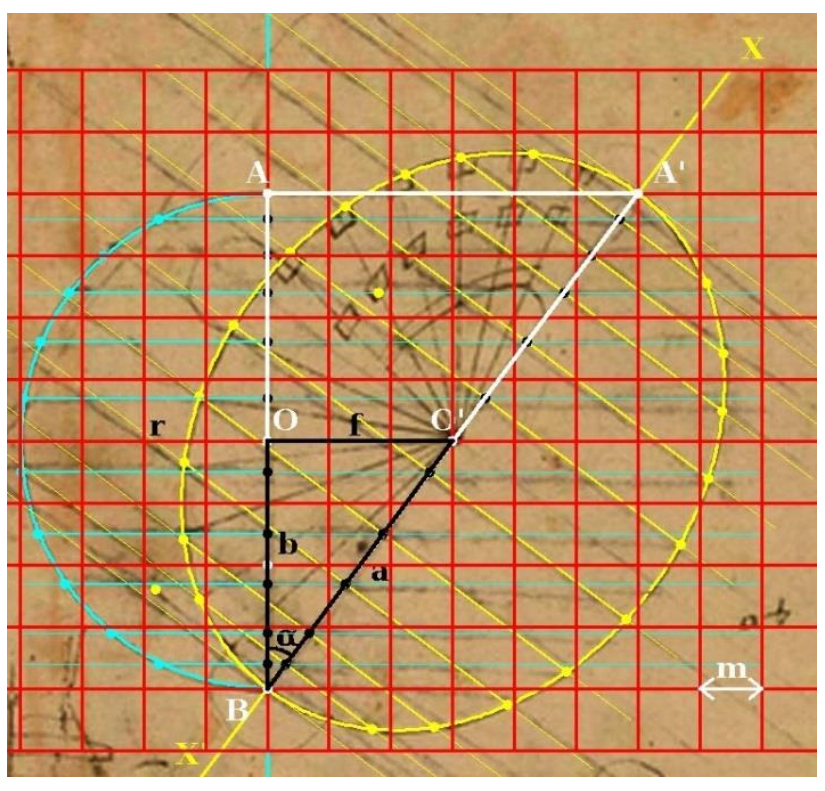

Figure 11. Construction de cette ellipse

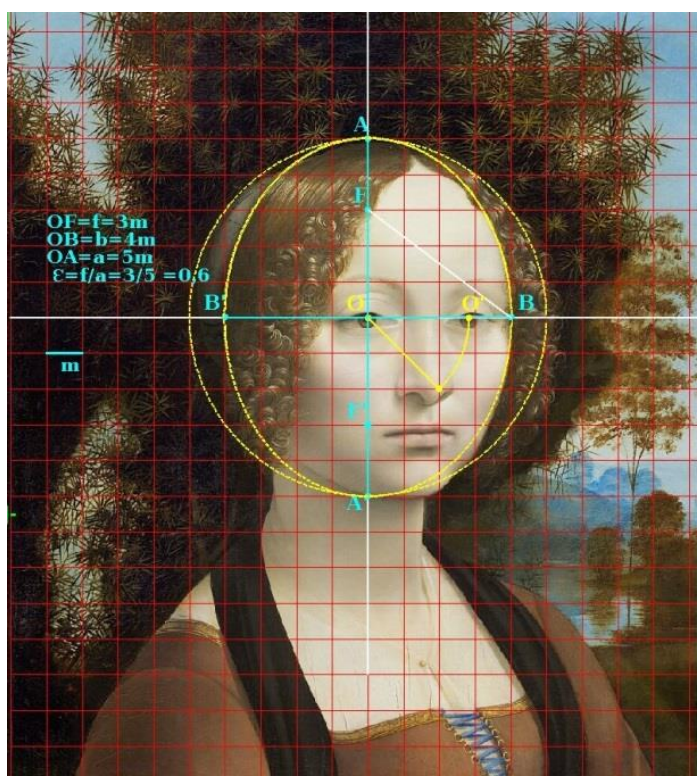

Figure 12. L'ellipse modélisant le visage de dans un maillage carré. Ginevra de’ Benci

Le triangle est facile à construire sur un maillage carré (figure 11). Soit $\boldsymbol{m}$ la largeur de la maille. Léonard a pris $\mathbf{A B}=8 \boldsymbol{m}, \mathbf{A} \mathbf{A}^{\prime}=6 \boldsymbol{m}$ et $\mathbf{A}^{\prime} \mathbf{B}=10 \boldsymbol{m}$. Par suite, les paramètres de l'ellipse sont les côtés du triangle rectangle $\mathbf{O O}^{\prime} \mathbf{B}: \mathbf{b}=\mathbf{r}=\mathbf{O B}=\mathbf{A B} / 2=4 m, \mathbf{a}=\mathbf{O}^{\prime} \mathbf{B}=\mathbf{A}^{\prime} \mathbf{B} / 2=5 m, \mathbf{f}=\mathbf{O O}^{\prime}=$ $\mathbf{A A}^{\prime} / 2=3 m$, et l'excentricité $\varepsilon=\mathbf{f} / \mathbf{a}=\sin \alpha=0,6$ et $\alpha=36^{\circ} \mathbf{5 2}$ '.

Il est intéressant de remarquer que l'ellipse du folio 602 possède la même excentricité $\boldsymbol{\varepsilon}=\mathbf{f} / \mathbf{a}=$ 0,6 que l'ellipse qui modélise (figure 12) une grande partie du visage ${ }^{10}$ de Ginevra de' Benci. Cette dernière ellipse a été construite sur un maillage carré avec les mêmes paramètres : $\mathbf{a}=5 \mathbf{m}, \mathbf{b}=4 \mathbf{m}$, $\mathbf{f}=3 \mathbf{m}$.

Toutefois, Léonard aurait pu aussi construire l'ellipse du visage de Ginevra de' Benci, en utilisant la méthode Df3, exposée figure 4a, et en prenant comme cercle principal le contour de la tête.

\subsection{Principe de construction de l'ellipse du folio 318 du Codex Atlanticus}

Le principe de construction de la forme elliptique du folio 318 du Codex Atlanticus, est très proche de celui qui a permis de construire selon la définition (Df4) la forme représentée sur le folio 602. Mais ici, Léonard a volontairement écarté l'ellipse du cercle principal (figure 13). 


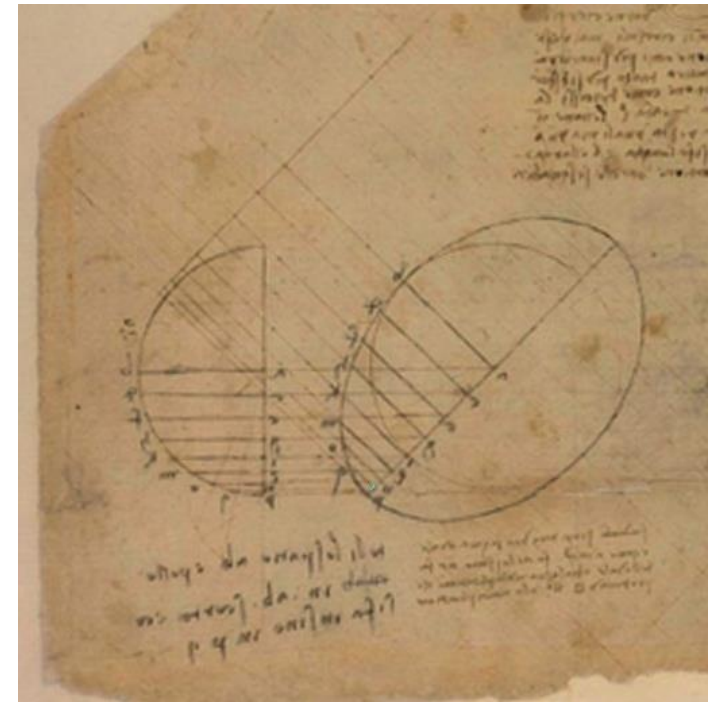

Figure 13. La forme elliptique du folio 318

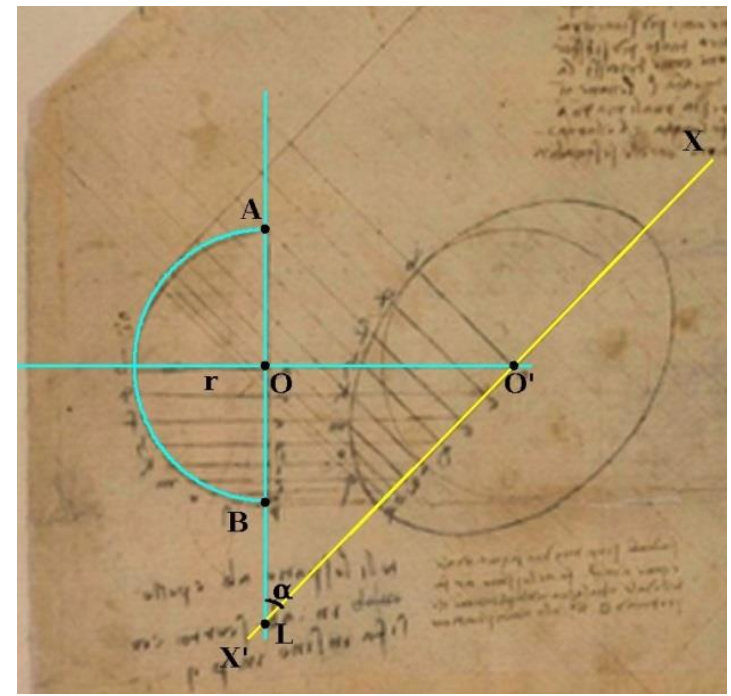

Figure 14. Les bases de la construction

De manière analogue au dessin du folio 602, Léonard a d'abord tracé (figure 14) un demi-cercle de centre $\mathbf{O}$ et de diamètre $\mathbf{A B}$. Puis il a mené un axe $\mathbf{X X}$ ', incliné d'un angle $\boldsymbol{\alpha}$ par rapport à l'axe vertical AB.

Il a ensuite mené (figure 15) un faisceau de droites horizontales, différemment espacées, traversant uniquement le quart de cercle inférieur, en formant des segments de droites qui sont de plus en plus rapprochés lorsqu'ils sont près du sommet $\mathbf{B}$. Ce faisceau de droites coupe l'axe (XX') en plusieurs points noirs. Il a reporté ces points par symétrie sur l'axe (XX') pour déterminer les points blancs. Par chacun de ces points noirs et blancs, il a fait passer une droite perpendiculaire à cet axe ( $\left.\mathbf{X X}^{\prime}\right)$, formant ainsi un autre faisceau de droites parallèles.

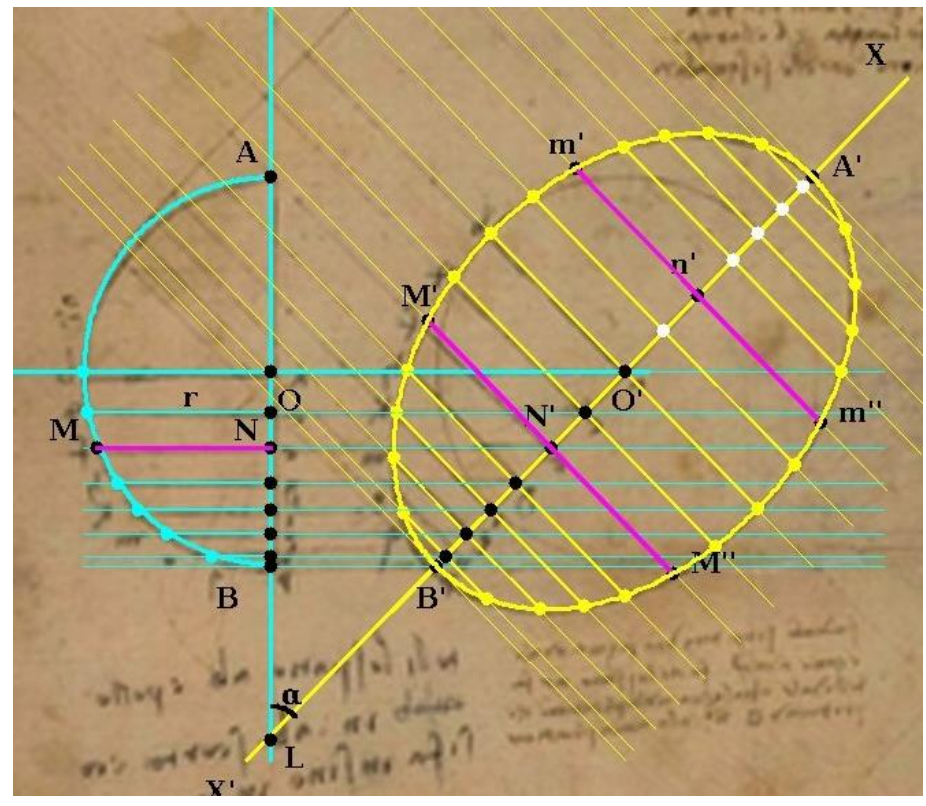

Figure 15. Construction de l'ellipse du folio 318.

Léonard a ensuite reporté 4 fois chacun des segments de droite définis par le faisceau horizontal $\mathrm{du}$ quart de cercle, sur les deux droites correspondantes perpendiculaires à l'axe (XX'), en respectant les symétries par rapport au grand axe et par rapport au petit axe. Par exemple le segment MN est reporté selon les segments M'N', N'M', m'n', n'm'. Il suffit alors de joindre leurs extrémités pour dessiner l'ellipse (figure 15). 
Le segment C'D' passant par O' correspond (figure 16) au petit axe de l'ellipse et le segment A'B' au grand axe. Par construction le demi-petit axe $\mathbf{b}$ de l'ellipse, formé par le segment de droite $\mathbf{O}^{\prime} \mathbf{C}^{\prime}$, est donc égal à $\mathbf{r}$. Le demi-grand axe a est tel que $\mathbf{r} / \mathbf{a}=\mathbf{O A} / \mathbf{O}^{\prime} \mathbf{A}^{\prime}=\cos (\boldsymbol{\alpha}), \operatorname{soit} \mathbf{a}=\mathbf{r} / \cos (\boldsymbol{\alpha})$.

\subsubsection{Choix de l'ellipse du folio 318 et sa construction dans un maillage carré}

Pour dessiner l'ellipse du folio 318, Léonard a encore choisi une ellipse particulière. Elle a été construite à partir de deux éléments : le quart de cercle de rayon $\mathbf{r}$ qui lui donne sa dimension et l'angle d'inclinaison $\boldsymbol{\alpha}$. Ces deux éléments sont contenus dans le triangle rectangle H'O'B' (figure 16). Ce triangle choisi par Léonard est un triangle rectangle isocèle. L'angle $\boldsymbol{\alpha}$ vaut $45^{\circ}$. Les côtés H'B' et H'O' sont égaux au rayon $\mathbf{r}$ du cercle. L'hypoténuse vaut $\mathbf{O}^{\prime} \mathbf{B}$ ' $=\mathbf{r} \sqrt{ } \mathbf{2}$.

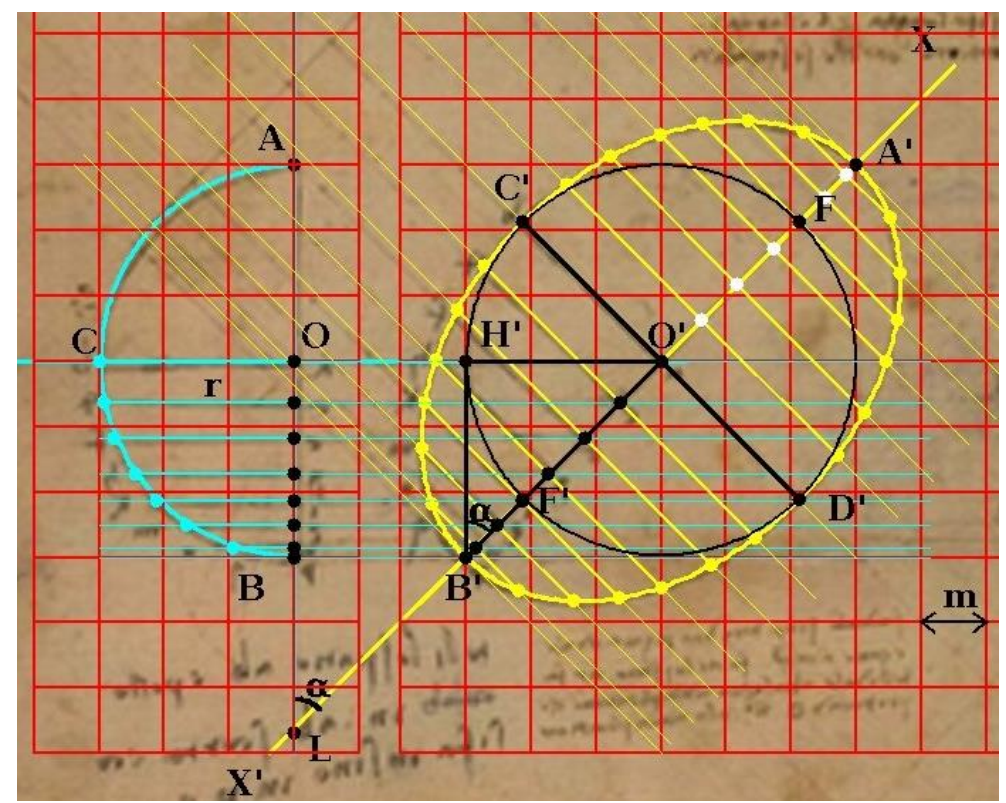

Figure 16. Construction de l'ellipse du folio 318 dans un maillage carré

Ce triangle peut être construit sur un maillage carré (figure 16). Soit $\boldsymbol{m}$ la largeur de la maille. Si nous prenons $\mathbf{H}^{\prime} \mathbf{B}^{\prime}=\mathbf{r}=3 m, \mathbf{O}^{\prime} \mathbf{B}^{\prime}=3 m \sqrt{ } \mathbf{2}$.

Les paramètres de l'ellipse sont alors : $\mathbf{a}=\mathbf{O}^{\prime} \mathbf{B}^{\prime}=3 \boldsymbol{m} \sqrt{ } \mathbf{2}, \mathbf{b}=\mathbf{O}^{\prime} \mathbf{C}^{\prime}=\mathbf{O}^{\prime} \mathbf{F}^{\prime}=\mathbf{f}=\mathbf{r}=3 \boldsymbol{m}$, et l'excentricité vaut $\boldsymbol{\varepsilon}=\mathbf{f} / \mathbf{a}=\sin (\alpha)=1 / \sqrt{ } \mathbf{2}$, avec $\boldsymbol{\alpha}=\mathbf{4 5}^{\circ}$.

Sur le folio 318, Léonard a tracé le petit cercle principal de rayon $\mathbf{r}$, centré au point $\mathbf{O}$ ', inscrit dans l'ellipse. Le cercle passe par les points $\mathbf{C}$ ' et $\mathbf{D}$ ' et par les deux foyers $\mathbf{F}$ et $\mathbf{F}$ '.

Il faut remarquer que cette ellipse particulière se retrouve dans plusieurs œuvres ${ }^{11}$ de Léonard : Salvator Mundi, La Dame à l'hermine, La Belle Princesse et le Portrait d'Isabelle d'Este. Ces œuvres présentent des contours assimilables à des arcs d'ellipse possédant la même excentricité $\varepsilon=$ $1 / \sqrt{ } 2$ que celle de l'ellipse du folio 318 . 


\subsection{Principe de construction de l'ellipse du folio 1032 du Codex Atlanticus}

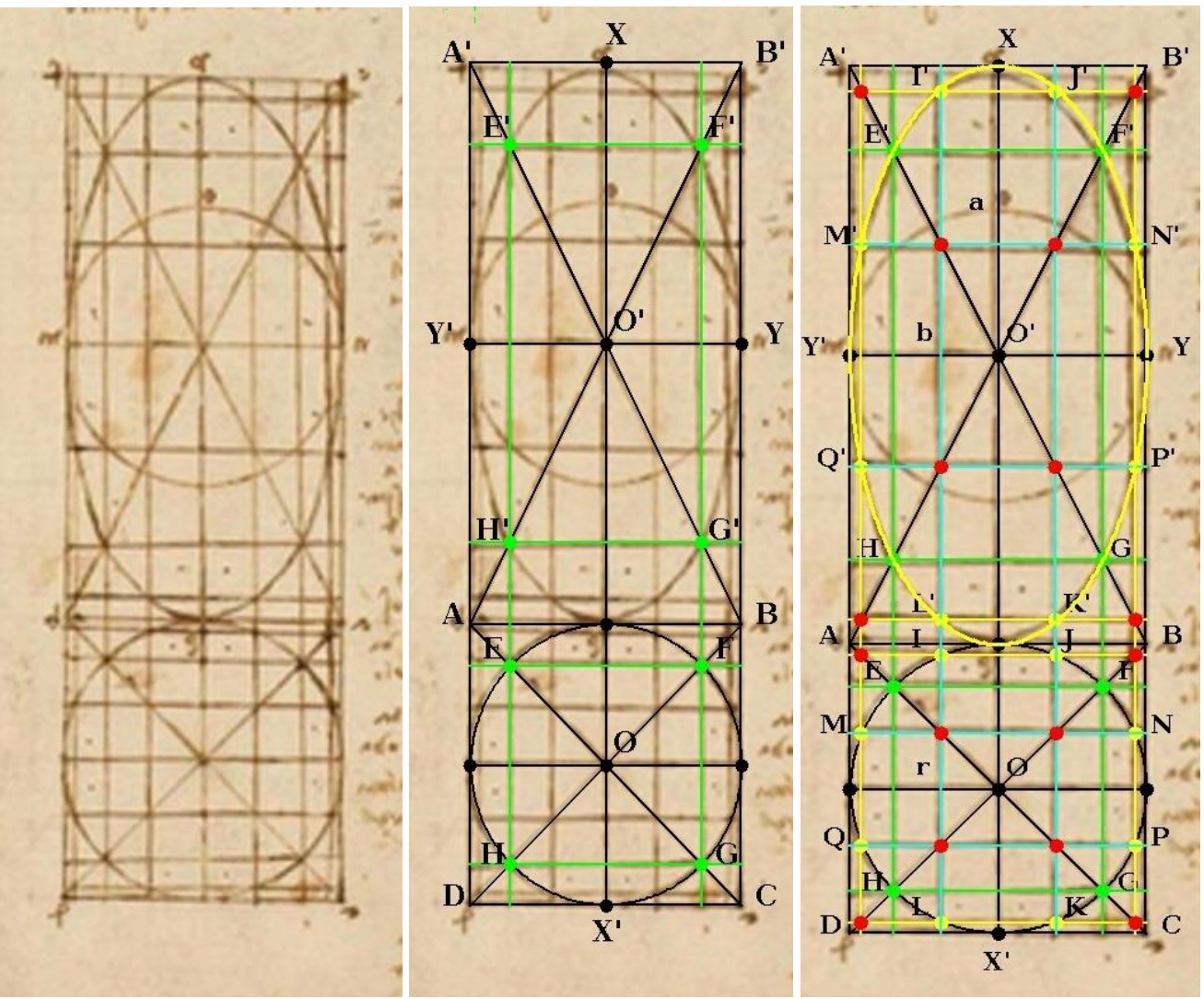

Figure 17a, b, c. Construction de l'ellipse du folio 1032

Afin d'éviter le report fastidieux des segments de droite dans la méthode de construction des ellipses exposée précédemment, Léonard propose dans le folio 1032 (1507) un autre principe de construction.

Ce principe est encore basé sur la projection d'un cercle sur un plan incliné : définition Df4. Comme précédemment, Léonard a placé le cercle principal et l'ellipse dans un même plan. Le cercle et l'ellipse centrés respectivement en $\mathbf{O}$ et $\mathbf{O}$ ', sont tous les deux situés sur un même axe $\mathbf{X}^{\prime} \mathbf{X}$. Le cercle est inscrit dans un carré de largeur 2r, et l'ellipse est inscrite dans un rectangle de la même largeur $2 \mathbf{r}$ et de longueur $\mathbf{l}=2 \mathbf{r} / \cos (\boldsymbol{\alpha})$. L'agrandissement $\mathbf{k}=1 / \cos (\boldsymbol{\alpha})$ est fonction de l'inclinaison.

Le demi-petit axe $\mathbf{b}$ de cette ellipse est égal au rayon $\mathbf{r}$ du cercle, et le demi-grand axe a est égal à $\mathbf{b} / \cos (\boldsymbol{\alpha})$.

Lors de cette projection, un point $\mathbf{M}$ de coordonnées $\left(\mathbf{x}_{\mathbf{c}}, \mathbf{y}_{\mathbf{c}}\right)$ du cercle se projette en un point $\mathbf{M}$ ' de coordonnées $\left(\mathbf{x}_{\mathbf{e}}, \mathbf{y}_{\mathrm{e}}\right)$ de l'ellipse avec $\mathbf{x}_{\mathrm{e}}=\mathbf{x}_{\mathbf{c}} / \cos (\boldsymbol{\alpha})$ et $\mathbf{y}_{\mathrm{e}}=\mathbf{y}_{\mathbf{c}}$.

Les ordonnées sont identiques, et les abscisses sont agrandies dans le rapport constant $1 / \cos (\boldsymbol{\alpha})$.

\subsubsection{Construction de l'ellipse du folio 1032}

Pour dessiner l'ellipse du folio 1032, Léonard a encore choisi une ellipse particulière, puisque l'ellipse est inscrite (figure 17a) dans un rectangle double carré, de telle sorte que $\mathbf{a}=2 \mathbf{b}, \cos (\boldsymbol{\alpha})=$ $1 / 2, \boldsymbol{\alpha}=60^{\circ}$, et $\varepsilon=\sin (\alpha)=0,866$.

Dans la partie basse du folio, Léonard a d'abord tracé le carré et le cercle inscrit, puis au-dessus, le rectangle double carré.

Les milieux des 4 côtés du rectangle donnent la position des quatre sommets de l'ellipse. 
Les diagonales du carré se projettent suivant les deux diagonales du rectangle, et leurs points d'intersection $\mathbf{E}, \mathbf{F}, \mathbf{G}, \mathbf{H}$ avec le cercle principal, se projettent suivant les points $\mathbf{E}$ ', F', G', H' appartenant à l'ellipse (figure 17 b).

Ensuite, utilisant les symétries du carré par rapport à l'axe vertical, et par rapport aux diagonales, Léonard trace à l'intérieur de ce carré (figure 17c) 4 droites (couleur cyan). Ces droites se coupent deux à deux avec les diagonales suivant 4 points rouges disposés en carré. Ces 4 droites coupent le cercle suivant les points $\mathbf{I}, \mathbf{J}, \mathbf{K}, \mathbf{L}, \mathbf{M}, \mathbf{N}, \mathbf{P}, \mathbf{Q}$. Les droites (couleur jaune) passant par ces 8 points se coupent deux à deux avec les diagonales suivant 4 autres points rouges disposés en carré.

Lors de la projection, les droites verticales se prolongent dans le rectangle, et les points rouges se projettent sur les diagonales de ce rectangle. Ces points déterminent la position des 4 droites horizontales dans ce rectangle qui correspondent aux 4 droites horizontales du carré. Les intersections de ces dernières avec les droites verticales définissent la position de 8 points I', J', K', L', M', N', P,' Q' qui appartiennent à l'ellipse et qui s'ajoutent aux 8 points précédents.

Il suffit maintenant de joindre ces 16 points pour tracer l'ellipse (figure 17c). Cette méthode utilisant les symétries du carré, est simple et précise. Elle ne nécessite pas de reports de segments. Comme le suggère Léonard, le cercle principal et le carré peuvent être centrés en un seul point $\mathbf{O}$ '.

Enfin, cette méthode peut se généraliser à la construction d'ellipses d'excentricité $\varepsilon=\sin (\alpha)$ différente. Il suffit, partant d'un cercle de rayon $\mathbf{r}$, de construire un rectangle dans la direction souhaitée, de largeur $2 \mathbf{r}$ et de longueur $\mathbf{l}=2 \mathbf{r} / \cos (\boldsymbol{\alpha})$.

\subsubsection{Construction de la forme elliptique du haut de la tête de La Dame à l'hermine.}

L'analyse géométrique interne (figure 18a) a montré ${ }^{12}$ que le haut de la tête de La Dame à l'hermine, présentait un contour qui pouvait être, en grande partie, modélisé, par une forme elliptique dont l'excentricité vaut $\boldsymbol{\varepsilon}=\mathbf{1} / \sqrt{ } \mathbf{2}$. Le grand axe est incliné d'un angle de $54^{\circ} 74$ par rapport à l'axe horizontal : cet angle est caractéristique du maillage harmonique puisqu'il correspond à la pente de la diagonale de la maille. Ce qui montre que l'ellipse a été tracée sur un maillage harmonique vertical dont la largeur et la hauteur de la maille ont pour valeurs respectives : $\boldsymbol{m}_{\boldsymbol{h}}$ et $\boldsymbol{m}_{v}$. Les paramètres de l'ellipse sont tels que $\mathbf{a}=\mathbf{b} \sqrt{2}$, avec $\mathbf{a}=2 \boldsymbol{m}_{v}$ et $\mathbf{b}=\mathbf{2} \boldsymbol{m}_{h}$.

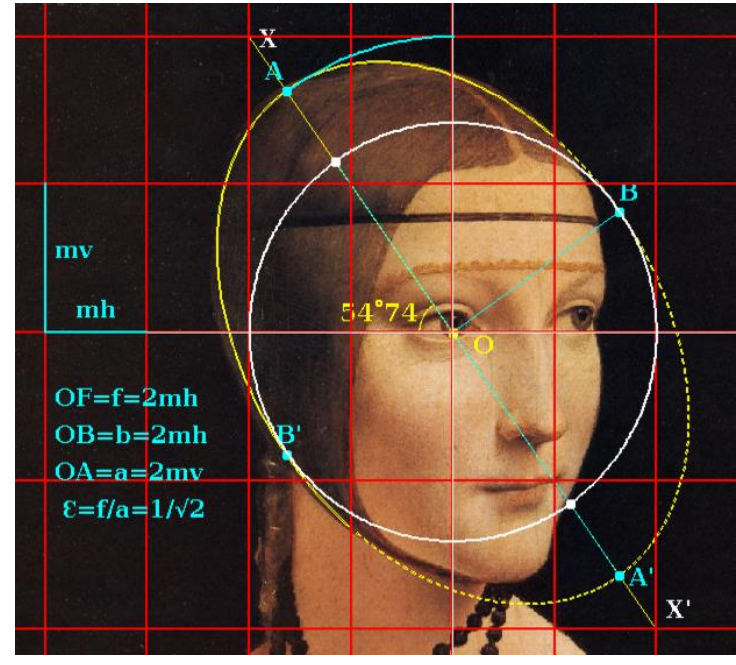

Figure 18a. Analyse géométrique du haut de la tête de La Dame à l'hermine

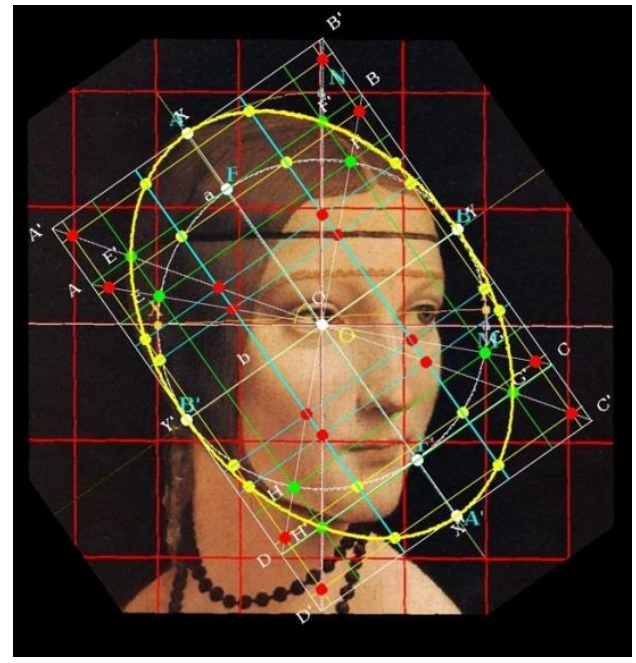

Figure 18b. Construction de la forme elliptique de la tête de La Dame à l'hermine 
Ce contour elliptique peut être retrouvé (figure 18b) en appliquant le principe de construction exposé ci-dessus. Il suffit, à partir du cercle de rayon $\mathbf{r}=\mathbf{b}=\mathbf{2} \boldsymbol{m}_{\boldsymbol{h}}$ centré en $\mathbf{O}$, de tracer dans la direction du grand axe $\mathbf{X}^{\prime} \mathbf{X}$, le carré $\mathbf{A B C D}$ dont le côté vaut $2 \mathbf{b}$, puis le rectangle $\mathbf{A}^{\prime} \mathbf{B}^{\prime} \mathbf{C}^{\prime} \mathbf{D}^{\prime}$ de même largeur et de longueur $2 \mathbf{a}=2 \mathbf{b} \sqrt{ } \mathbf{2}$. C'est un rectangle harmonique. Les milieux des côtés de ce rectangle déterminent les 4 sommets de l'ellipse. Les diagonales du carré se projettent suivant les diagonales du rectangle, et les 4 points d'intersection $\mathbf{E}, \mathbf{F}, \mathbf{G}, \mathbf{H}$ avec le cercle principal, se projettent suivant les 4 points d'intersection $\mathbf{E}^{\prime}, \mathbf{F}^{\prime}, \mathbf{G}^{\prime}, \mathbf{H}^{\prime}$ avec l'ellipse. Enfin comme il a été décrit ci-dessus, les intersections de 4 droites verticales avec 4 droites horizontales dont la position est déterminée par la projection, définissent 8 autres points appartenant à l'ellipse. Il suffit maintenant de joindre ces 16 points pour tracer l'ellipse de la tête de la Dame à l'hermine

\subsection{Superposition d'un maillage carré et d'un maillage harmonique}

Dans le cas particulier où l'on cherche à construire une ellipse d'excentricité $\boldsymbol{\varepsilon}=\mathbf{1} / \sqrt{\mathbf{2}}$, qui est d'ailleurs la forme elliptique que l'on rencontre le plus souvent dans les œuvres de Léonard, le principe de construction de l'ellipse du folio 1032 nous a suggéré une méthode originale que Léonard a peut-être utilisée ou qu'il aurait pu utiliser. Cette ellipse a pour excentricité $\boldsymbol{\varepsilon}=\mathbf{1} / \sqrt{ } \mathbf{2}, \boldsymbol{\alpha}=$ $45^{\circ}$, et un facteur de grandissement $\mathrm{k}=\mathbf{1} / \cos (\boldsymbol{\alpha})=\sqrt{ } \mathbf{2}$. Il suffit donc d'agrandir les abscisses des points du cercle, d'un facteur $\mathrm{k}=\sqrt{\mathbf{2}}$, pour obtenir les points correspondants de l'ellipse.

\subsubsection{Méthode des deux maillages superposés.}
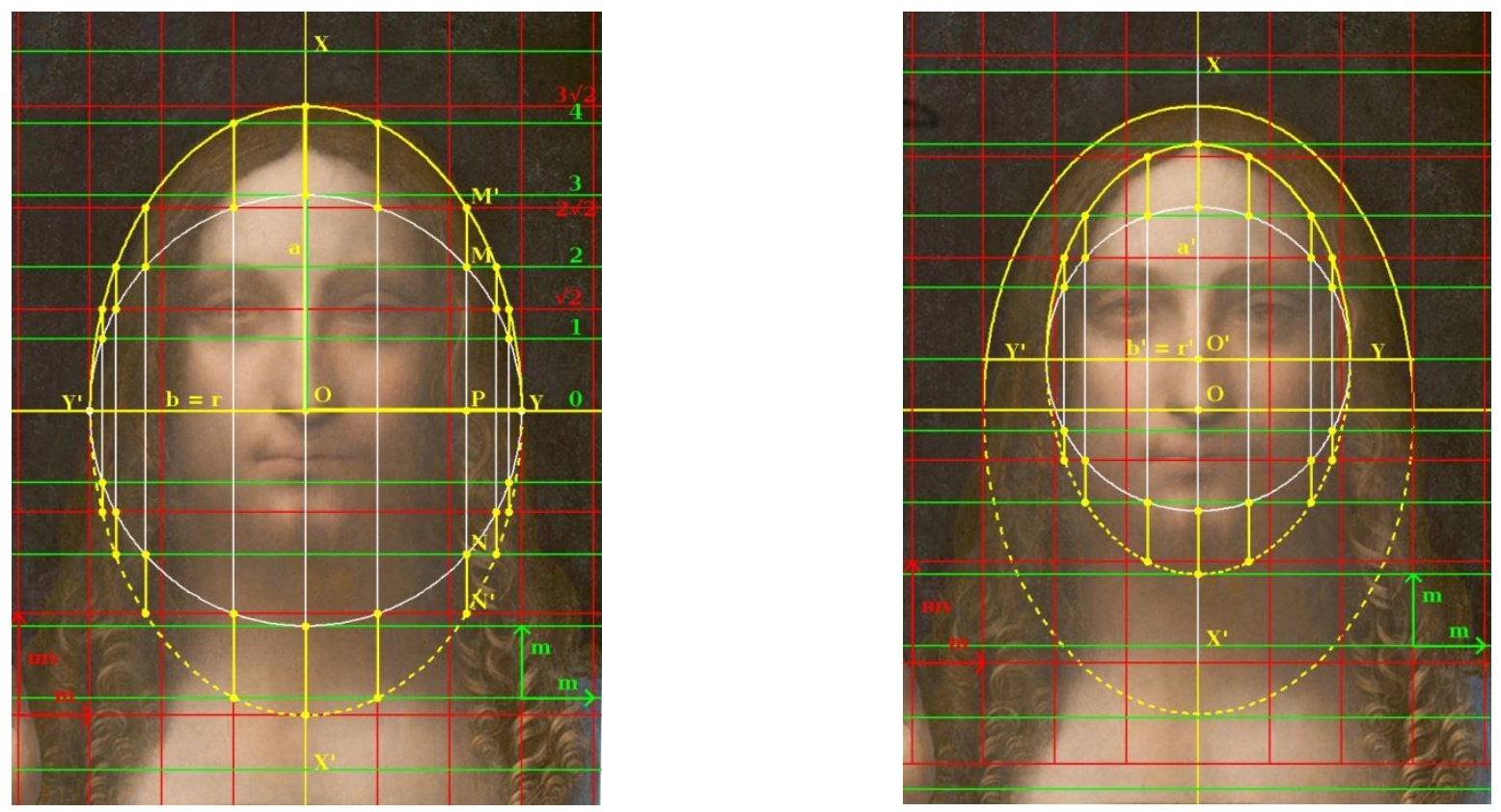

Figure 19. Méthode des deux maillages superposés :

a. Construction de la forme elliptique modélisant le haut de la tête du Salvator Mundi

b. Construction de la forme elliptique modélisant le haut du visage du Salvator Mundi

Le principe consiste à utiliser deux maillages superposés, tous les deux centrés en $\mathbf{O}$ : un maillage carré (lignes vertes) dont la maille a pour côté $\boldsymbol{m}$, et un maillage harmonique (lignes rouges) dont la maille a la même largeur $\boldsymbol{m}$ et une hauteur $\boldsymbol{m}_{\boldsymbol{v}}=\boldsymbol{m} \sqrt{\mathbf{2}}$. Pour illustrer cette méthode, nous montrons comment elle permet de construire la forme elliptique (figure 19a) qui modélise le haut de la tête du Salvator Mundi ${ }^{13}$.

Soit $\mathbf{M}$, le point d'intersection d'une ligne horizontale d'un maillage et du cercle. Le point $\mathbf{M}$ a pour ordonnée $\mathbf{y}_{\mathbf{M}}=\mathbf{O P}$ et pour abscisse $\mathbf{x}_{\mathbf{M}}=\mathbf{P M}$. Le point $\mathbf{M}$ se projette sur le point $\mathbf{M}^{\prime}$ de 
coordonnées : $\mathbf{y}_{\mathbf{M}},=\mathbf{y}_{\mathbf{M}}$ et $\mathbf{x}_{\mathbf{M}},=\mathbf{x}_{\mathbf{M}} \sqrt{ } \mathbf{2}$, qui est situé sur la ligne horizontale de l'autre maillage et appartenant à l'ellipse. Par exemple, (figure 19a) $\mathbf{x}_{\mathbf{M}}=2 \boldsymbol{m}$ et $\mathbf{x}_{\mathbf{M}},=2 \boldsymbol{m} \sqrt{2}$.

Ainsi à chaque intersection $\mathbf{M}$ du cercle par une ligne horizontale d'un maillage, correspond un point M' situé sur la ligne horizontale de l'autre maillage, de telle sorte que $\mathbf{x}_{\mathbf{M}}$ ' $=\mathbf{x}_{\mathbf{M}} \sqrt{ } 2$. Par exemple si $\mathbf{x}_{M}=2 \boldsymbol{m} \sqrt{2}, \mathbf{x}_{M},=4 \boldsymbol{m}$, et si $\mathbf{x}_{M}=-\boldsymbol{m} \sqrt{2}, \mathbf{x}_{M^{\prime}},=-2 \boldsymbol{m}$.

En donnant aux maillages une largeur de maille $\boldsymbol{m}$, telle que le rayon du cercle $\mathbf{r}=\mathbf{b}=3 \boldsymbol{m}$, nous obtenons avec la symétrie, 18 points, appartenant à l'ellipse. En particulier, au centre $: \mathbf{y}_{\mathbf{M}}=0, \mathbf{x}_{\mathbf{M}}=$ $\mathbf{b}=3 \boldsymbol{m}$, et $\mathbf{x}_{\mathbf{M}},=\mathbf{a}=3 \boldsymbol{m} \sqrt{ } 2$. Il suffit de joindre tous ces points ainsi obtenus, pour réaliser le tracé de l'ellipse (figure 19a) ou de joindre seulement les points qui correspondent à la forme elliptique recherchée pour le haut de la tête du Salvator Mundi.

En décalant les maillages vers le haut, d'une hauteur $\boldsymbol{m}$, et en prenant un cercle principal de rayon $\mathbf{r}^{\prime}=3 m / \sqrt{ } 2$, nous obtenons en appliquant la même méthode (figure 19b), 16 points appartenant à une nouvelle ellipse. En particulier, au centre $\mathbf{y}_{\mathbf{M}}=0, \mathbf{x}_{\mathbf{M}}=\mathbf{b}^{\prime}=3 \boldsymbol{m} / \sqrt{2}$, et $\mathbf{x}_{\mathbf{M}^{\prime}}=\mathbf{a}^{\prime}=3 \boldsymbol{m}$. Il suffit de joindre ces points pour obtenir la forme elliptique ${ }^{14} \mathrm{du}$ contour du haut du visage du Salvator Mundi.

Dans cette méthode applicable aux ellipses de type 1, il n'y a ni report de segments, ni tracé de lignes de construction. Seuls les agrandissements des abscisses des points d'intersection des droites du maillage et du cercle, déterminent les points permettant le tracé de l'ellipse. Ces derniers $\boldsymbol{\varepsilon}=\mathbf{1} / \sqrt{ } \mathbf{2}$ définissent (figures 19) deux croissants de lune ${ }^{15}$ qui mettent bien en évidence la projection du cercle sur un plan incliné.

\subsubsection{Construction de la forme elliptique de la tête de La Dame à l'hermine à l'aide la méthode des deux maillages superposés}
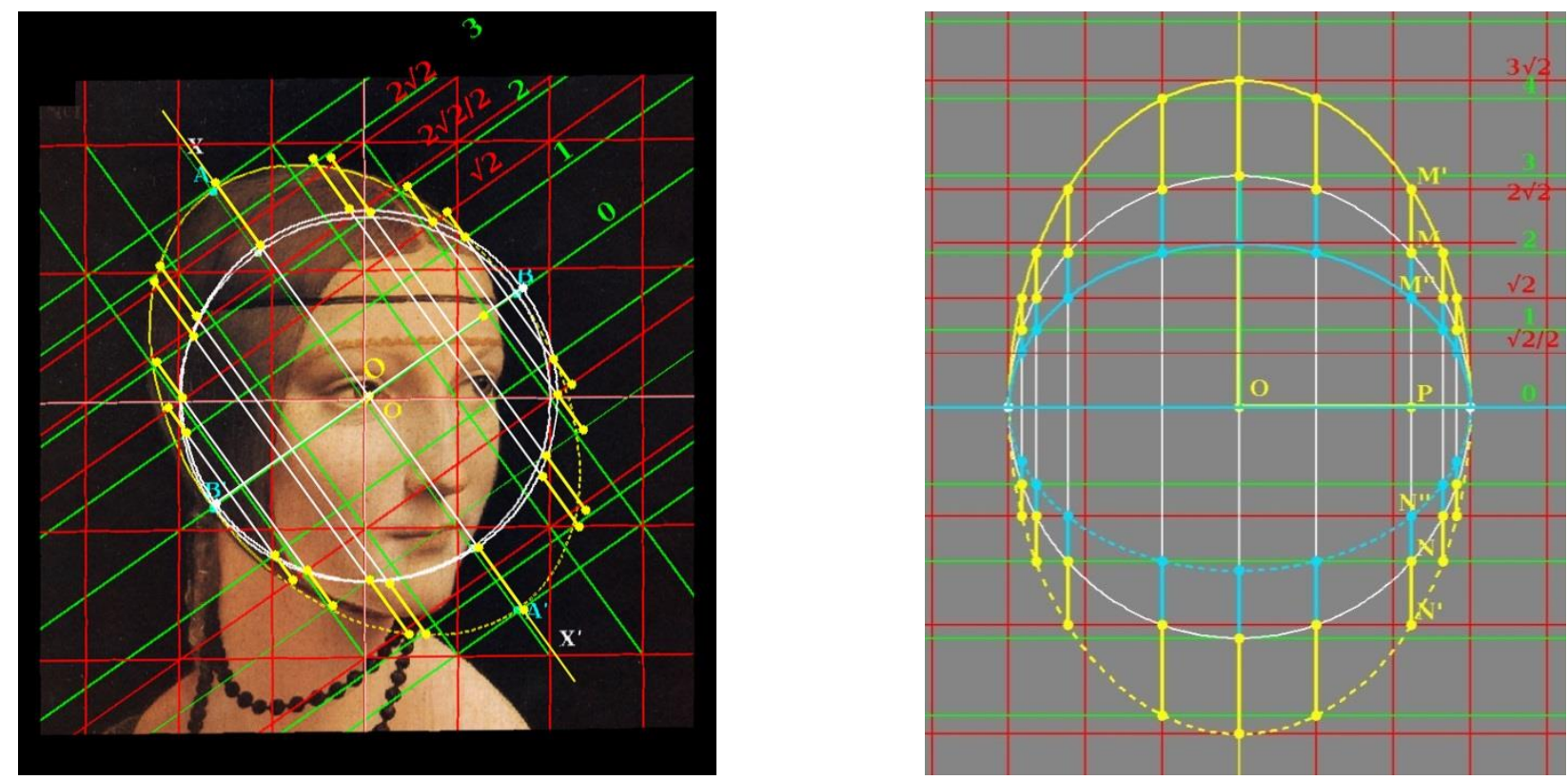

Figure 20. Méthode des deux maillages superposés

a. Autre construction de la forme elliptique du haut de la tête de La Dame à l'hermine

b. Construction d'une forme elliptique orthogonale à la première.

\footnotetext{
${ }^{14}$ Les ellipses de la tête et du visage du Salvator ont même excentricité. La seconde est V2 fois plus petite que la première : $\mathbf{a}^{\prime} / \mathbf{a}=\mathbf{b}^{\prime} / \mathbf{b}=\mathbf{1} / \mathbf{V} \mathbf{2}$. Sa surface est donc deux fois plus petite. Elles sont consonantes, et résonnent à l'octave.

${ }^{15}$ Une lunule est une portion de surface délimitée par deux cercles non cncentriques de rayons différents. 
Comme nous l'avons rappelé ci-dessus, le haut de la tête de La Dame à l'hermine (1488, 1490), présente un contour qui peut être en grande partie modélisé par un arc d'ellipse dont l'excentricité ${ }^{16}$ $\boldsymbol{\varepsilon}=\mathbf{1} / \sqrt{ } \mathbf{2}$. Nous pouvons donc réaliser sa construction par la méthode exposée ci-dessus. A partir du centre $\mathbf{O}$, et en s'appuyant sur le maillage harmonique existant (figure 18a), il faut tracer (figure 20a) dans la direction de l'axe $\mathbf{X}^{\prime} \mathbf{X}$, deux maillages obliques superposés : un maillage carré (lignes vertes) dont la maille a pour côté $\boldsymbol{m}$ ', et un maillage harmonique (lignes rouges) dont la maille possède la même largeur $\boldsymbol{m}$ ' et une hauteur $\boldsymbol{m}^{\prime}{ }_{v}=\boldsymbol{m}{ }^{\prime} \sqrt{\mathbf{2}}$. À chaque intersection $\mathbf{M}$ du cercle par une ligne horizontale d'un maillage, correspond un point $\mathbf{M}$ ' situé sur la ligne horizontale de l'autre maillage, de telle sorte que $\mathbf{x}_{\mathbf{M}},=\mathbf{x}_{\mathbf{M}} \sqrt{2}$. Nous obtenons ainsi 18 points appartenant à l'ellipse. Il suffit de réunir ces points pour obtenir le contour elliptique du haut de la tête de La dame à l'hermine.

\subsubsection{Remarque}

Dans cette méthode des deux maillages superposés, si au lieu d'augmenter les abscisses des points d'intersection des droites du maillage avec le cercle, nous diminuons ces abscisses d'un facteur $\mathrm{k}=\sqrt{\mathbf{2}}$, nous obtenons grâce aux deux maillages, un même nombre de points qui correspondent encore à une ellipse, mais celle-ci est située horizontalement (figure 20b). Le demigrand axe a' est égal à $\mathbf{b}$ et le demi-petit axe $\mathbf{b}^{\prime}$ 'est égal à $\mathbf{b} / \sqrt{ } \mathbf{2}$, de telle sorte que $\mathbf{a} / / \mathbf{b}^{\prime}=\mathbf{a} / \mathbf{b}=\sqrt{\mathbf{2}}$. C'est à nouveau une ellipse d'excentricité $\varepsilon=1 / \sqrt{ } \mathbf{2}$. Elle est précisément égale à la forme elliptique modélisant le haut du visage du Salvator Mundi. On remarquera que cette dernière transformation correspond, selon la définition Df3, à la projection orthogonale sur un plan horizontal d'un cercle situé dans un plan incliné, (figure 4b), ou de façon plus imagée, à l'intersection d'un cylindre incliné par un plan horizontal.

\section{Discussion}

Cette étude, basée sur les connaissances trigonométriques actuelles, montre que le principe de construction des ellipses utilisé par Léonard pour ces trois dessins, est tout a fait correct. Cependant les dessins sont incomplets. Léonard n'a représenté ni les foyers, ni même la distance focale $\mathbf{f}$, que nous avons représentés et évalués pour faciliter la compréhension. Il n'a recours ni à la première définition de l'ellipse : Df1, ni à la méthode du jardinier : Df2. Il ne semble pas considérer l'ellipse comme une conique, mais simplement comme une élongation régulière du cerle, c'est-à-dire comme la projection du cercle sur un plan incliné.

\subsection{Définition de l'inclinaison.}

Léonard n'a pas non plus indiqué l'angle d'inclinaison $\boldsymbol{\alpha}$ qui est pourtant caractéristique de l'ellipse, car il ignorait probablement les fonctions trigonométriques, et les tables trigonométriques ne seront publiées qu'en 1571 par François Viète dans son livre : Canon Mathematicus. Léonard ne caractérisait pas l'inclinaison comme un angle exprimé en degrés ou en radians, mais comme le rapport ${ }^{17} \mathbf{A B} / \mathbf{O A}$ entre deux côtés d'un triangle rectangle tracé sur cet angle (figure 21). Ce rapport correspond de nos jours au sinus de l'angle $\boldsymbol{\alpha}$. Il était plus significatif pour Léonard d'exprimer $1^{\prime}$ inclinaison du folio 602 par le rapport $3 / 5$, que par un angle de $36^{\circ} 52^{\prime}$ '.

\footnotetext{
${ }^{16}$ Voir plus loin : Table 1.

${ }^{17}$ Cette manière de mesurer un angle par le rapport de la longueur de deux segments, est propre aux géomètres de l'Antiquité, elle est en accord avec l'étymologie même du mot trigonométrie qui provient du grec trigônos, triangle, et métron, mesure. Elle demeure encore aujourd'hui, puisqu'on évalue la montée d'une côte, non pas en degrés mais en pourcent (par exemple 3 


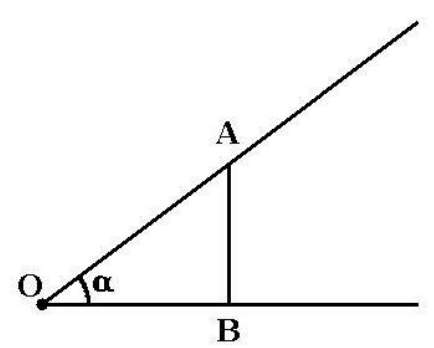

Figure 21. Définition de l'inclinaison

Enfin si l'axe d'inclinaison est représenté sur le folio 318 (figure 13), il n'est pas tracé sur le folio 602 (figure 7).

Pour construire une ellipse, Léonard commençait par établir un petit triangle rectangle qui lui donnait la valeur du sinus et par suite l'excentricité $\varepsilon=\sin (\alpha)$. Ainsi, comme nous l'avons montré, il a défini l'excentricité du dessin du folio 602 à l'aide du triangle rectangle $(3,4,5)$ OO'B (figure 11), et celle du dessin du folio 318, à l'aide du triangle rectangle isocèle H'O'B' (figure 16).

Bien plus, si l'on donne à $\mathbf{O B}$ la valeur $\mathbf{b}, \mathbf{O A}$ a pour valeur $\mathbf{a}$, et $\mathbf{A B}$ a pour valeur $\mathbf{f}$. Ces trois paramètres vérifient la relation de Pythagore $: \mathbf{a}^{2}=\mathbf{b}^{2}+\mathbf{f}^{2}$.

\subsection{Classification des ellipses}

Ainsi, puisque la construction de l'ellipse est liée à la forme du petit triangle rectangle, nous avons cherché quels étaient les petits triangles rectangles que Léonard pouvait facilement construire sur un maillage carré ou sur un maillage harmonique, et déterminer ainsi l'excentricité recherchée.

Nous avons classé ces petits triangles rectangles suivant la valeur AB/OA (correspondant au sinus de l'angle), et indiqué les ellipses correspondantes dans deux tables : la première (table 1) pour les valeurs du sinus inférieures ou égales à $\mathbf{1} / \sqrt{\mathbf{2}}$, et la seconde (table 2) pour les valeurs supérieures ou égales à $\mathbf{1} / \sqrt{ } \mathbf{2}$. La première correspond aux ellipses arrondies, la seconde aux ellipses allongées.

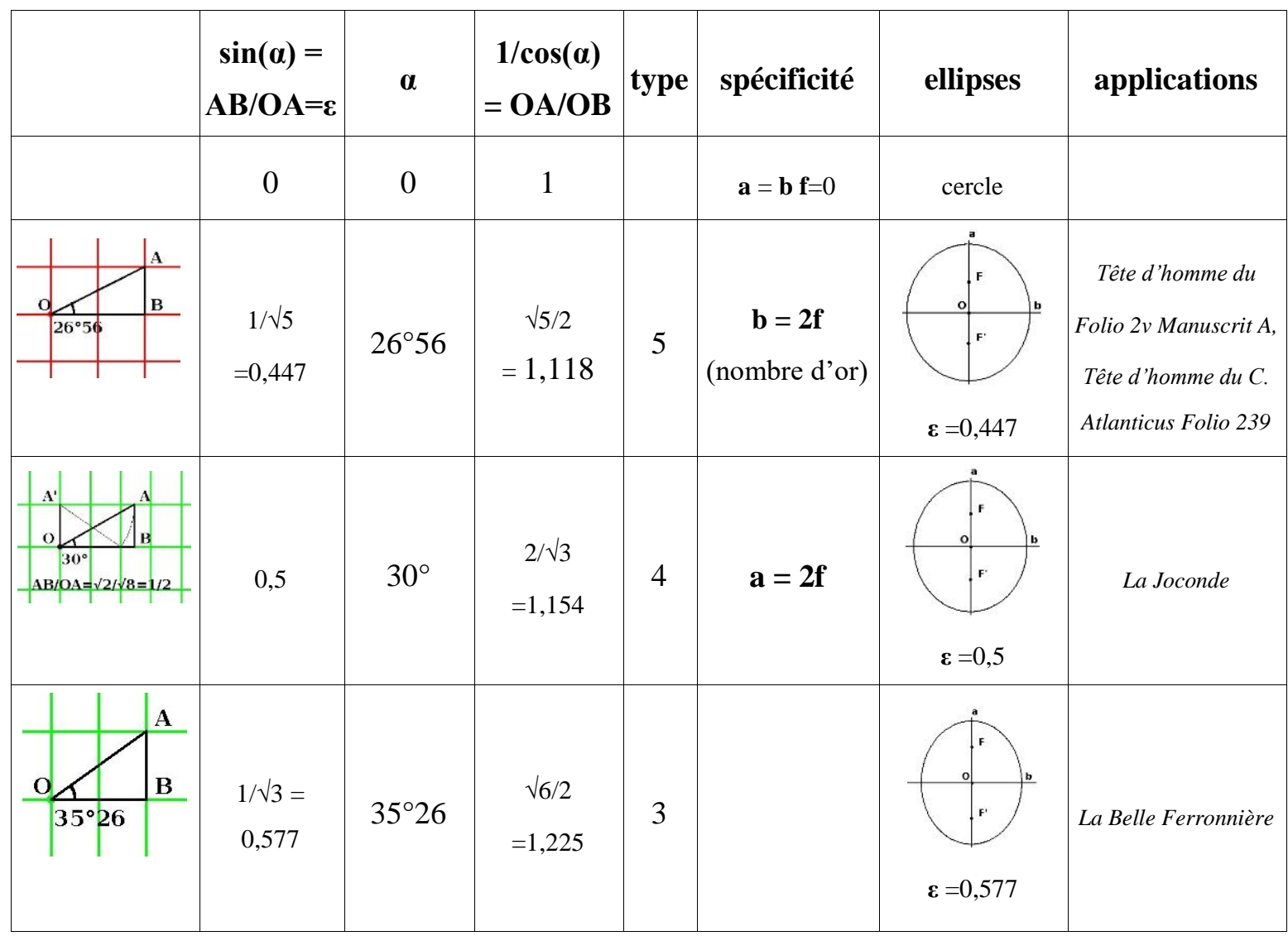




\begin{tabular}{|c|c|c|c|c|c|c|c|}
\hline 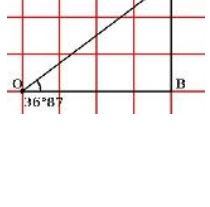 & 0,6 & $36^{\circ} 87$ & $\begin{array}{c}5 / 4 \\
=1,25\end{array}$ & $2 a$ & $\begin{array}{c}\mathbf{f}, \mathbf{b}, \mathbf{a} / / / 3, \\
4,5\end{array}$ & $\varepsilon=0,6$ & $\begin{array}{l}\text { Ginevra de'Benci, } \\
\text { Codex Atlanticus } \\
\text { folio } 602\end{array}$ \\
\hline 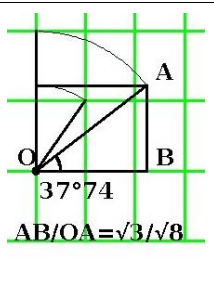 & $\begin{aligned} & \sqrt{ }(3 / 8) \\
= & 0,612\end{aligned}$ & $37^{\circ} 74$ & $\begin{array}{l}\sqrt{ } 8 / \sqrt{ } 5 \\
=1,265\end{array}$ & 2 & $\begin{array}{c}\mathbf{f}, \mathbf{b}, \mathbf{a} \\
/ / / \sqrt{ } 3, \sqrt{ } 5, \sqrt{ } 8\end{array}$ & $\boldsymbol{\varepsilon}=0,612$ & \\
\hline 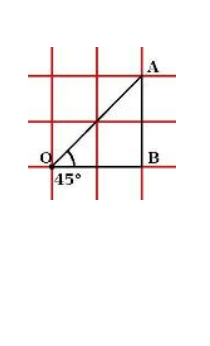 & $\begin{aligned} & 1 / \sqrt{ } 2 \\
= & 0,707\end{aligned}$ & $45^{\circ}$ & $\begin{array}{c}\sqrt{ } 2 \\
=1,414\end{array}$ & 1 & $\mathbf{f}=\mathbf{b}$ & $\boldsymbol{\varepsilon}=0,707$ & $\begin{array}{l}\text { Salvator Mundi, } \\
\text { La Dame à l'hermine, } \\
\text { La Belle Princesse, } \\
\text { Isabelle d'Este, } \\
\text { Codex Atlanticus } \\
\text { folio } 318 .\end{array}$ \\
\hline
\end{tabular}

Table 1. Table des ellipses plutôt arrondies en fonction de l'inclinaison $\sin (\alpha)$.

Les colonnes de la table indiquent successivement : la construction du petit triangle rectangle sur un maillage carré (rouge) ou sur un maillage harmonique (vert), la valeur de $\sin (\alpha)$, l'angle d'inclinaison $\alpha$, l'agrandissement des segments $O A / O B=1 / \cos (\alpha)$, le type de l'ellipse, ses particularités, la forme de l'ellipse pour un a constant, et le nom du portrait ou du dessin de Léonard, dont le contour présente partiellement la forme elliptique correspondante.

\begin{tabular}{|c|c|c|c|c|c|c|c|}
\hline & $\begin{array}{c}\sin \left(\alpha^{\prime}\right) \\
\mathrm{AB} / \mathrm{OA}=\varepsilon^{\prime}\end{array}$ & $\alpha '$ & $\begin{array}{l}1 / \cos \left(\alpha^{\prime}\right) \\
=\text { OA/OB }\end{array}$ & type & spécificité & ellipses & applications \\
\hline \begin{tabular}{l|l|l} 
& & $A$ \\
& & \\
$\alpha$ & & \\
$\alpha 5^{\circ}$ & & \\
\end{tabular} & $\begin{aligned} & 1 / \sqrt{ } 2 \\
= & 0,707\end{aligned}$ & $45^{\circ}$ & $\begin{array}{c}\sqrt{ } 2 \\
=1,414\end{array}$ & 1 & $\mathbf{f}=\mathbf{b}$ & $\varepsilon^{\prime}=0,707$ & $\begin{array}{l}\text { Salvator Mundi, } \\
\text { La Dame à l'hermine, } \\
\text { La Belle Princesse } \\
\text { Isabelle d'Este } \\
\text { Codex Atlanticus } \\
\text { folio } 318\end{array}$ \\
\hline 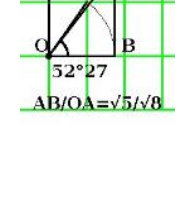 & $\begin{aligned} & \sqrt{ } 2 / \sqrt{ } 3 \\
= & 0,791\end{aligned}$ & $52^{\circ} 27$ & $\begin{aligned} & \sqrt{ } 8 / \sqrt{ } 3 \\
= & 1,633\end{aligned}$ & $2^{\prime}$ & $\begin{array}{c}\mathbf{b , f}, \mathbf{a} \\
/ / / \sqrt{ } 3, \sqrt{ } 5, \sqrt{ } 8\end{array}$ & $\begin{array}{c}\boldsymbol{\varepsilon}^{\prime}=0,791\end{array}$ & \\
\hline 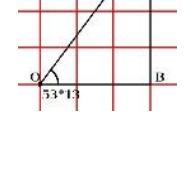 & 0,8 & $53^{\circ} 13$ & $\begin{aligned} & 5 / 3 \\
= & 1,666\end{aligned}$ & $2 a^{\prime}$ & $\begin{array}{c}\mathbf{f}, \mathbf{b}, \mathbf{a} \\
/ / / 4,3,5\end{array}$ & $\boldsymbol{\varepsilon}^{\prime}=0,8$ & \\
\hline
\end{tabular}




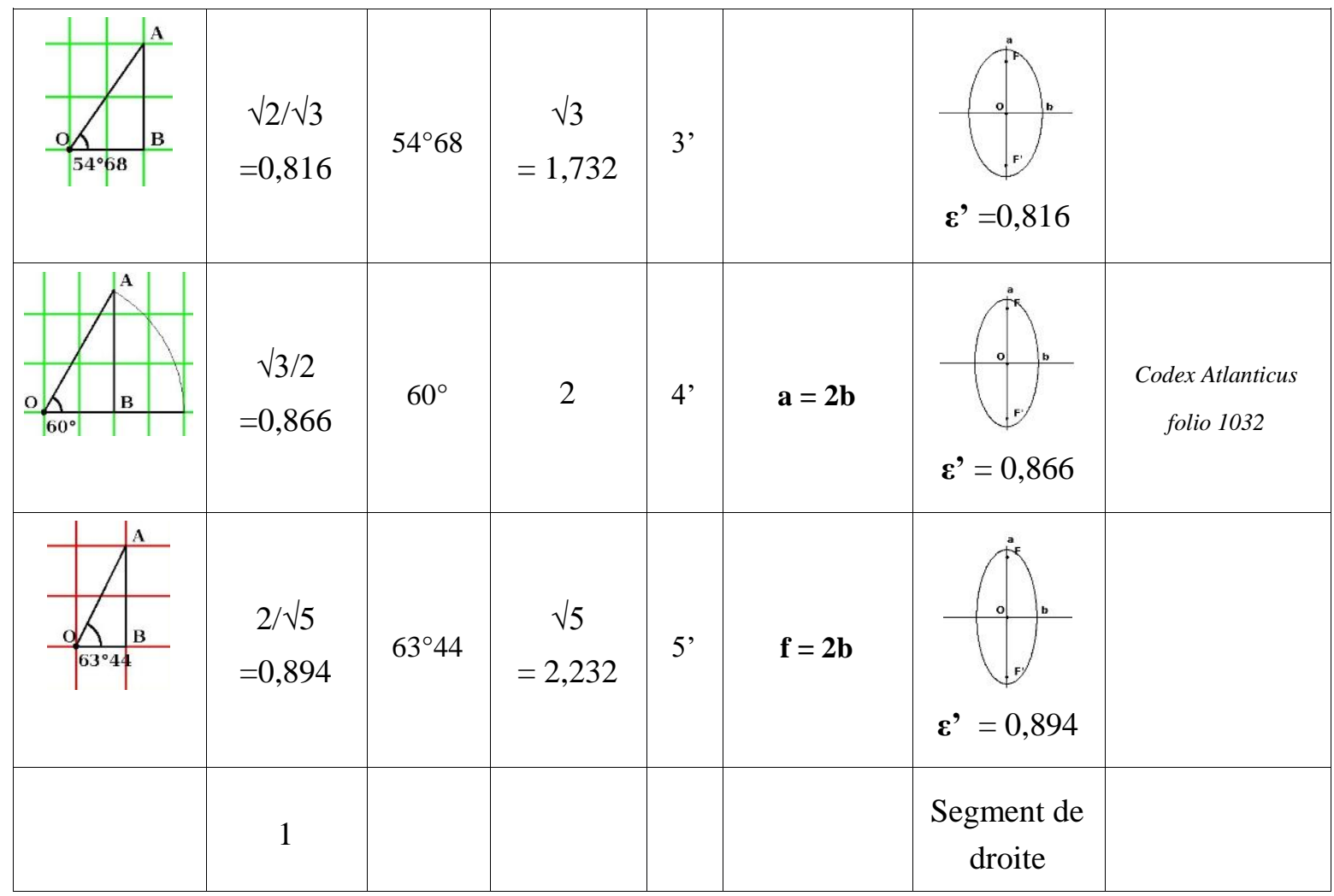

Table 2. Table des ellipses plutôt allongées en fonction de l'inclinaison $\sin \left(\alpha^{\prime}\right)$.

Les colonnes de la table indiquent successivement : la construction du petit triangle rectangle sur un maillage carré (rouge) ou sur un maillage harmonique (vert), la valeur de sin( $\left.\boldsymbol{\alpha}^{\prime}\right)$, l'angle d'inclinaison $\boldsymbol{\alpha}^{\prime}$, l'agrandissement des segments $O A / O B=1 / \cos \left(\alpha^{\prime}\right)$, le type de l'ellipse, ses particularités, la forme de l'ellipse pour un a' constant, et le nom du portrait ou du dessin de Léonard, dont le contour présente partiellement la forme elliptique correspondante.

Comme nous l'avions pressenti ${ }^{18}$, le nombre d'ellipses pouvant être construites par Léonard et par les peintres de cette époque est relativement petit (environ une douzaine). La plupart sont des ellipses particulières puisqu'elles présentent chacune, une relation particulière entreleurs paramètres. A chaque ellipse de la table 1 correspond une ellipse de la table 2. Ces deux ellipses sont complémentaires, leurs angles d'inclinaison sont tels que : $\boldsymbol{\alpha}+\boldsymbol{\alpha}^{\prime}=\mathbf{9 0}^{\circ}$. Elles possèdent les mêmes paramètres, mais les paramètres $\mathbf{b}$ et $\mathbf{f}$ sont permutés. Leurs excentricités $\boldsymbol{\varepsilon}$ et $\boldsymbol{\varepsilon}$ 'vérifient la relation : $\varepsilon^{2}+\varepsilon^{2}=1$.

\section{Tracé continu des ellipses par un procédé mécanique}

Pour passer du cercle à l'ellipse, Léonard, a cherché dans ces trois folios, une solution géométrique (qu'il n'a cessé d'améliorer) afin de déterminer les points particuliers permettant le tracé de l'ellipse à main levée.

Quelques années plus tard, afin d'éviter les erreurs induites par le dessin à main levée, Léonard a probablement imaginé un instrument (le compas à coniques ${ }^{19}$ ) qui permet de construire mécaniquement la solution. «Leonardo da Vinci a également utilisé des procédés mécaniques pour le tracé continu des coniques.... Vinci a représenté un compas parabolique dans le Codex

\footnotetext{
${ }^{18}$ Crettez J-P.,[3], §. 2.4.4.

${ }^{19}$ Carlos Pedretti lui en attribue la paternité dans Studi vinciani (Genève 1957) 
Atlanticus, folio 1093 a (1515) (figure 22). Ce dessin explicite un schéma plus ancien que l'on trouve dans le Codex Arundel, folio 73 r (1509) (figure 23)... ${ }^{20}{ }_{»}$

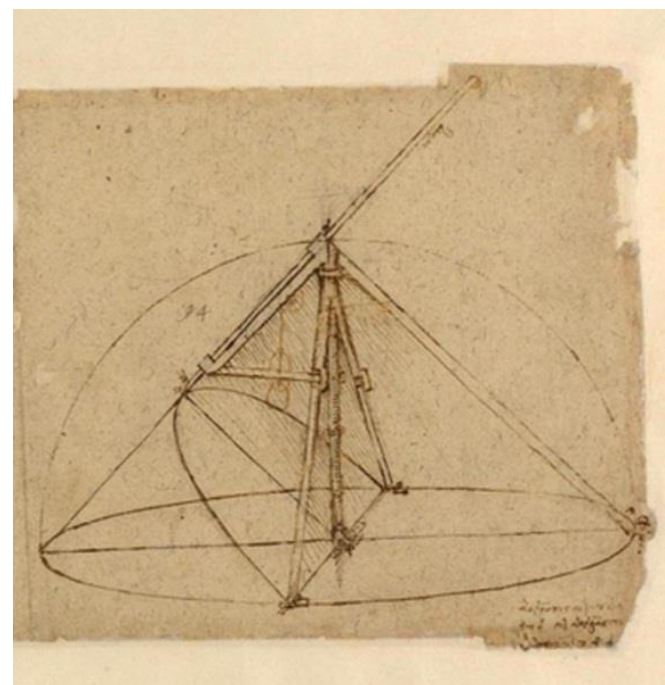

Figure 22. Schéma du compas parabolique parabolographe) : Codex Atlanticus folio 1093 (1515).

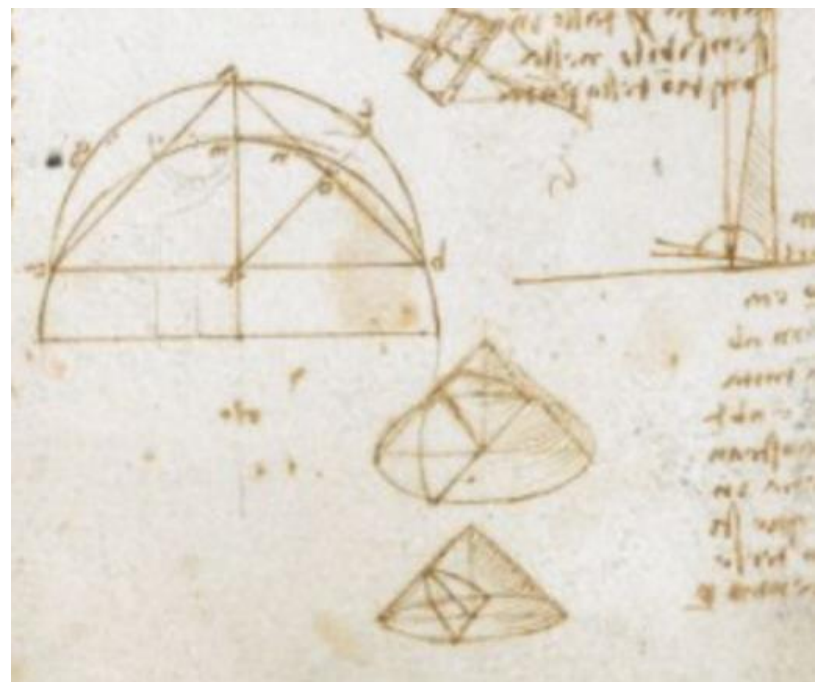

Figure 23. Dessin du principe du compas parabolique : Codex Arundel, folio 73r (1509).

\subsection{Principe du compas à coniques}

Le compas à coniques (seste da far l'ouato) (figure 24) parfois appelé compas parfait, permet de tracer la courbe, représentant l'intersection d'un cône incliné par un plan horizontal (P). Il est composé de trois branches qui assurent sa stabilité.

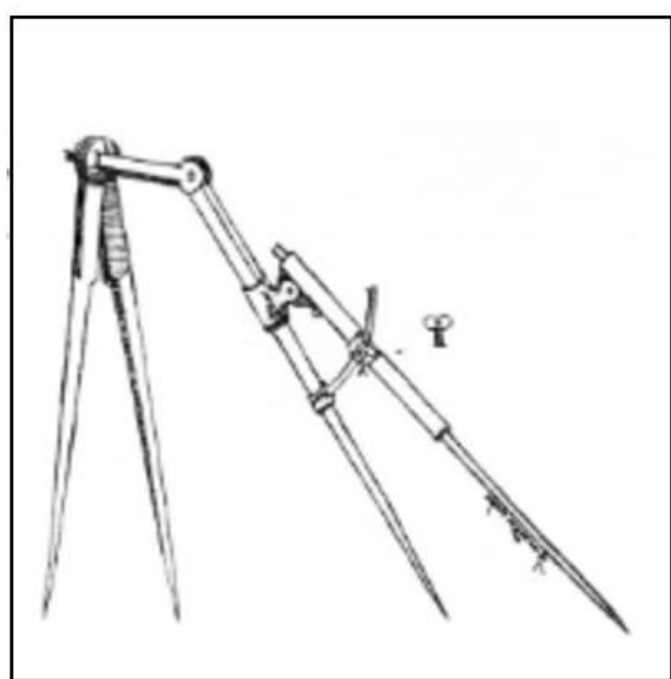

Figure 24. Schéma du compas à coniques Volpaia $^{21}$

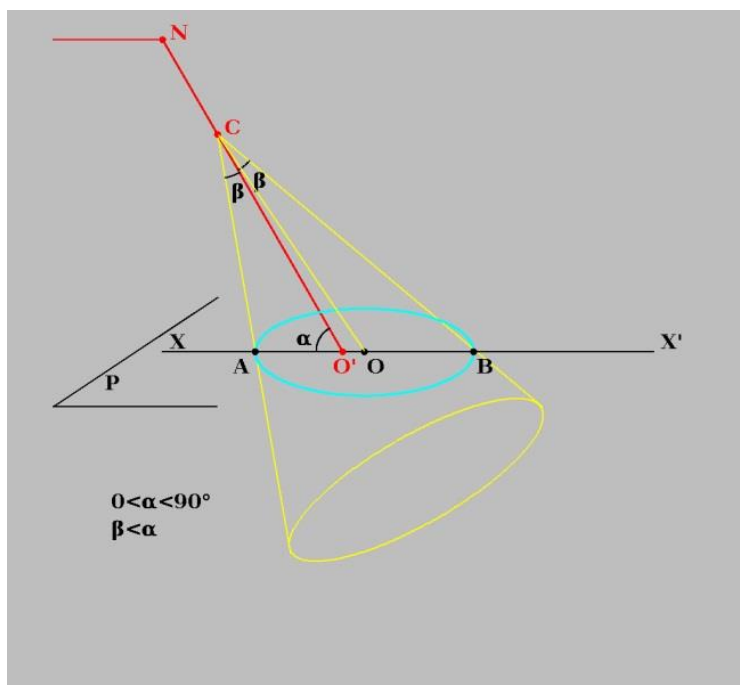

Figure 25. Construction d'une ellipse à l'aide d’après della du compas à coniques.

La branche de droite NO' (figure 25) représente l'axe du cône. Elle se termine par une pointe sèche. La branche est inclinée d'un angle $\boldsymbol{\alpha}$ par rapport au plan horizontal.

\footnotetext{
${ }^{20}$ Dominique Raynaud [7], Le tracé continu des sections coniques à la Renaissance.

${ }^{21}$ Benvenuto della Volpaia, horloger, instrumentiste à Florence (1486-1532) 
Un bras CB fixé au point $\mathbf{C}$, en faisant un angle $\boldsymbol{\beta}$, peut tourner autour de l'axe NO'. Il porte un tire-ligne télescopique qui peut glisser à l'intérieur de ce bras. En tournant et en glissant, "Questa va in su e in giu (tire-ligne). Questo gira (axe)», la pointe de ce tire-ligne peut ainsi balayer la surface de révolution du cône, dont l'angle au sommet est égal à $2 \boldsymbol{\beta}$. Ainsi, en s'appuyant sur le plan et en tournant, la pointe de ce tire-ligne trace la courbe intersection de ce cône avec le plan.

\subsection{Principe de l'intersection d'un cône incliné par un plan horizontal}

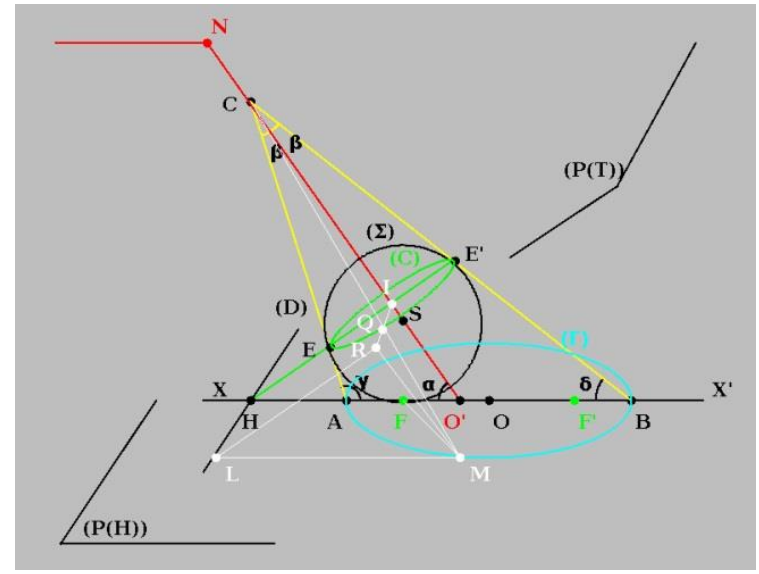

Figure 26. Intersection d'un cône incliné par plan horizontal

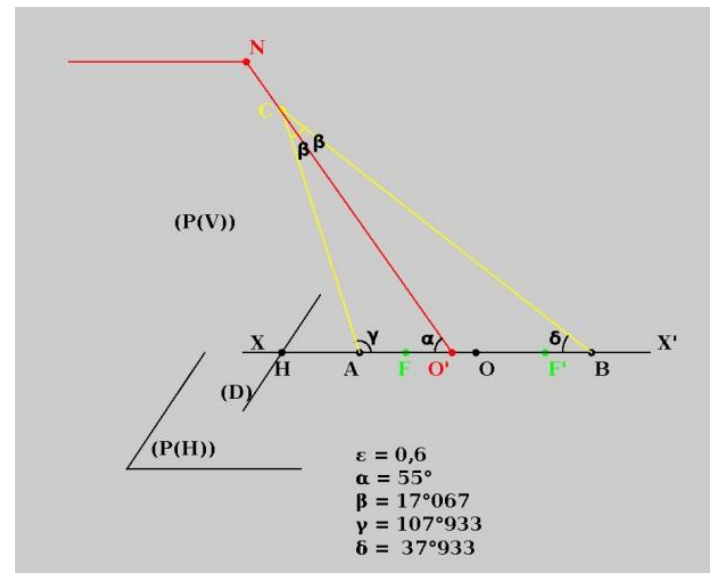

Figure 27. Détermination de la position O' de la pointe sèche

Dans l'intersection d'un cône incliné de sommet $\mathbf{C}$ par un plan horizontal $\mathbf{P}(\mathbf{H})$, il existe (figure 26) une sphère $(\boldsymbol{\Sigma})$, et une seule, de centre $\mathbf{S}$, inscrite dans le cône, tangente en un point $\mathbf{F}$ au plan $\mathbf{P}(\mathbf{H})$, et tangente à l'intérieur du cône le long d'un cercle $\mathbf{( C )}$ qui est situé dans le plan transversal $\mathbf{P}(\mathbf{T})$ perpendiculaire à l'axe du cône. Soit (D) la droite d'intersection des plans $\mathbf{P}(\mathbf{H})$ et $\mathbf{P}(\mathbf{T})$.

Le rapport des distances d'un point arbitraire $\mathbf{M}$ du plan $\mathbf{P}(\mathbf{H})$ au plan $\mathbf{P}(\mathbf{T})$ et à la droite $(\mathbf{D})$, a pour valeur : $\mathbf{M R} / \mathbf{M L}=\cos (\boldsymbol{\alpha})$. D'autre part, le cône est le lieu des points $\mathbf{M}$ de l'espace tels que : $\mathbf{M R} / \mathbf{M Q}=\cos (\boldsymbol{\beta})$. Ainsi, dans le plan $\mathbf{P}(\mathbf{H})$, la courbe $(\boldsymbol{\Gamma})$ intersection du cône par ce plan est le lieu des points tels que : MQ/ML $=\cos (\boldsymbol{\alpha}) / \cos (\boldsymbol{\beta})$.

Mais comme les longueurs des tangentes $\mathbf{M Q}$ et $\mathbf{M F}$ à la sphère $(\boldsymbol{\Sigma})$ issues du même point $\mathbf{M}$, sont égales, la courbe $(\boldsymbol{\Gamma})$ est le lieu des points $\mathbf{M}$ du plan $\mathbf{P}(\mathbf{H})$ dont le rapport $\mathbf{M F} / \mathbf{M L}$ des distances au point $\mathbf{F}$ et à la droite $(\mathbf{D})$ a une valeur constante $\cos (\boldsymbol{\alpha}) / \cos (\boldsymbol{\beta})$. C'est donc une conique de foyer $\mathbf{F}$, de directrice $(\mathbf{D})$, et d'excentricité $\varepsilon=\cos (\boldsymbol{\alpha}) / \cos (\boldsymbol{\beta})$.

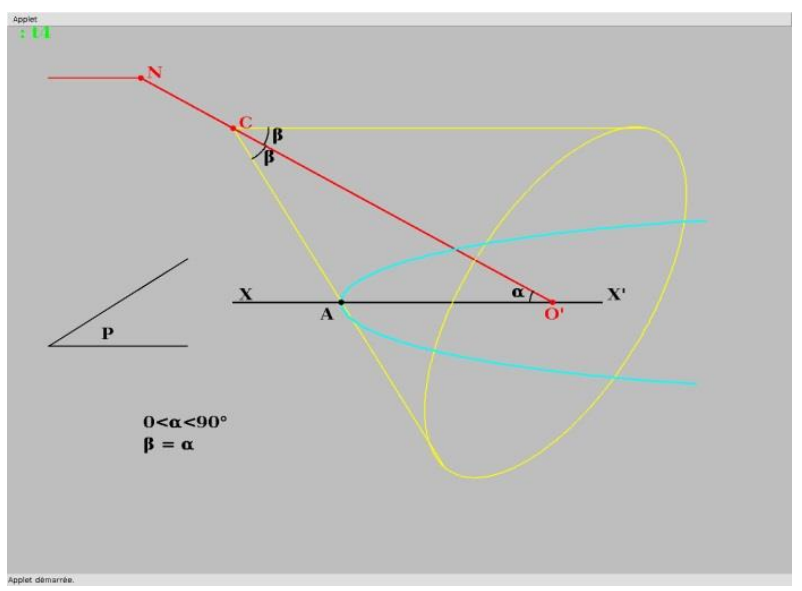

Figure 28. Construction d'une parabole par le compas à coniques

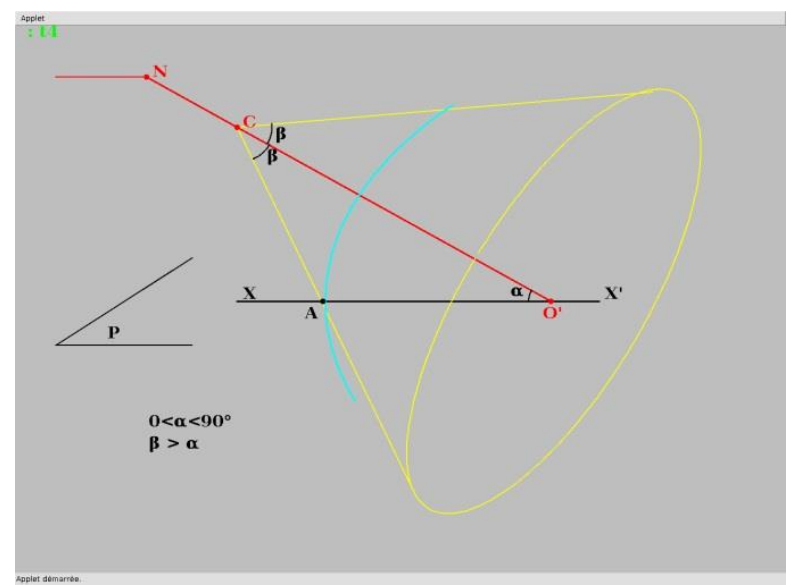

Figure 29. Construction d'une demi-branche d'hyperbole par le compas à coniques 
- Lorsque l'excentricité $\boldsymbol{\varepsilon}$ est comprise entre 0 et 1 , l'angle $\boldsymbol{\beta}$ est inférieur à l'angle d'inclinaison $\boldsymbol{\alpha}$ (figure 25), l'intersection est une ellipse selon la définition Df1.

- Lorsque l'excentricité $\boldsymbol{\varepsilon}$ est égale à 1, l'angle $\boldsymbol{\beta}$ est égal à l'angle d'inclinaison $\boldsymbol{\alpha}$ (figure 28), l'intersection est une parabole, d'axe $\mathbf{X Y}$ et de sommet $\mathbf{A}$.

- Enfin, lorsque l'excentricité $\boldsymbol{\varepsilon}$ est supérieure à 1, l'angle $\boldsymbol{\beta}$ est supérieur à l'angle d'inclinaison $\boldsymbol{\alpha}$ (figure 29), l'intersection est une demi branche d'hyperbole de sommet $\mathbf{A}$.

\subsection{Application du compas à ellipse}

Il est important de remarquer (figure 24) que le centre $\mathbf{O}$ de l'ellipse est différent du point $\mathbf{O}$ ' pied de la pointe sèche. Léonard désirant tracer une ellipse de centre $\mathbf{O}$, de grand axe $\mathbf{A B}$ et d'excentricité donnée $\boldsymbol{\varepsilon}$, devait ouvrir le compas avec des angles $\boldsymbol{\alpha}$ et $\boldsymbol{\beta}$ liés par la relation $\boldsymbol{\varepsilon}=$ $\cos (\boldsymbol{\alpha}) / \cos (\boldsymbol{\beta})$, puis déterminer la position $\mathbf{O}^{\prime}$ sur laquelle pointe le bras fixe. La première méthode pour choisir O', consiste à procéder par approximations successives. L'autre méthode est basée sur les angles (figure 27) : la génératrice passant par $\mathbf{A}$ selon l'angle $\boldsymbol{\gamma}=\left(\mathbf{1 8 0}^{\circ}-(\boldsymbol{\alpha}+\boldsymbol{\beta})\right)$, et la génératrice passant par $\mathbf{B}$ selon l'angle $\boldsymbol{\delta}=(\boldsymbol{\alpha}-\boldsymbol{\beta})$, se rencontrent au sommet $\mathbf{C}$ du cône. L'axe de ce cône, représenté dans le plan vertical $\mathbf{P}(\mathbf{V})$ par la bissectrice du triangle $\mathbf{A C B}$, coupe l'axe $\mathbf{X X}^{\prime}$ 'au point $\mathbf{O}$ '. Le schéma (figure 27) représente le compas à coniques paramétré pour tracer une ellipse de même excentricité $\varepsilon=0,6$ que celle qui modélise le visage de Ginevra de’ Benci.

\section{Conclusion}

Lorsqu'il effectuait un portrait, Léonard commençait par faire l'esquisse de la tête du personnage en traçant l'ellipse constituant la forme première. L'ellipse représentait pour lui, une courbe géométriquement pure comme celle du cercle, mais présentant une courbure permettant de s'adapter aux formes naturelles, résultantes temporaires des lois de la mouvance du monde.

Plus ingénieur que théoricien, Léonard ne formulant aucune théorie, enseignant le comment des solutions plus que le pourquoi, a commencé, comme le montre la datation des trois folios, par effectuer le tracé de l'ellipse par points, bien avant de rechercher à effectuer son tracé continu.

Ces trois constructions et celle que nous avons proposée, sont basées sur le principe de la projection sur un plan incliné, d'un cercle placé dans un plan horizontal. Mais à chaque fois, cette projection spatiale est transposée dans un même plan : celui du dessin. Léonard détermine la position exacte de certains points de la courbe, avant de les relier à main levée avec toute sa dextérité.

Dans ces trois dessins, Léonard ne représente ni les foyers, ni même la distance focale f. Il n'exprime pas l'excentricité par $\boldsymbol{\varepsilon}=\mathbf{f} / \mathbf{a}$, mais simplement par $\boldsymbol{\varepsilon}=\sin (\boldsymbol{\alpha})$. Ne s'intéressant pas aux foyers, il n'utilise pas la méthode du jardinier : (définition Df2). Il considère l'ellipse comme l'élongation du cercle, exactement comme l'ombre d'une pièce d'un florin sur un plan incliné.

Léonard ne mesure pas cette inclinaison par l'angle $\alpha$, mais par le sinus de cet angle ; c'est-à -dire le rapport entre deux côtés d'un triangle rectangle tracé sur cet angle : ce qui le conduit à ne considérer pratiquement qu'un petit nombre d'ellipses.

Ce n'est que vers les années 1509 , qu'il propose le compas à coniques pour effectuer un tracé continu de cette forme elliptique.

Le commentaire de Daniel Arasse relatif à la démarche de Léonard pour résoudre le problème dit «d'Alhazen » peut aussi s'appliquer à son approche du tracé des formes elliptiques : «La science n'est pas, pour lui, un objet de contemplation mais l'outil d'une action et son attitude est, le plus souvent, celle d'un ingénieur: il cherche des solutions pratiques qui, s'il le faut, sont atteintes à 
l'aide d'instruments mécaniques. Ne parvenant pas, malgré de multiples essais, à en trouver la solution géométrique, Léonard imagine un instrument qui en construit mécaniquement la solution $^{22}$.»

\section{Bibliographie}

[1] Arasse D., Léonard de Vinci, Edition Hazan, Paris, 1993.

[2] Clarck K., Léonard de Vinci, librairie générale Française, 2008.

[3] Crettez J-P., Les supports de la géométrie interne des peintres : de Cimabue à G. de La Tour. Editions ISTE (2017).

[4] Crettez J-P., Openscience -Géométrie interne du "Salvator Mundi" (version dite Cook, attribuée à Léonard de Vinci)

[5] Crettez J-P., Openscience -D'un simple dessin de Léonard de Vinci aux "formes premières".

[6] Kemp M., Leonardo, Oxford, University Press, 2005.

[7] Raynaud D. Le tracé continu des sections coniques à la Renaissance : Applications optico-perspectives, héritage de la tradition mathématique arabe. Arabic Sciences and Philosophy, CambridgeUniversity Press (CUP), 2007, 17, pp.299-345. 